\title{
Stable learning of functional maps in self-organizing spiking neural networks with continuous synaptic plasticity
}

\author{
Narayan Srinivasa* and Qin Jiang \\ Center for Neural and Emergent Systems, HRL Laboratories LLC, Malibu, CA, USA
}

\section{Edited by:}

Cornelius Glackin, University of Hertfordshire, UK

Reviewed by:

James A. Bednar, University of Edinburgh, UK

Cees Van Leeuwen, Katholieke Universiteit Leuven, Belgium

\section{${ }^{*}$ Correspondence:}

Narayan Srinivasa, Center for Neural and Emergent Systems, HRL Laboratories LLC, 3011 Malibu Canyon Road, Malibu, CA 91388, USA.

e-mail: nsrinivasa@hrl.com
This study describes a spiking model that self-organizes for stable formation and maintenance of orientation and ocular dominance maps in the visual cortex (V1). This self-organization process simulates three development phases: an early experience-independent phase, a late experience-independent phase and a subsequent refinement phase during which experience acts to shape the map properties. The ocular dominance maps that emerge accommodate the two sets of monocular inputs that arise from the lateral geniculate nucleus (LGN) to layer 4 of $\mathrm{V} 1$. The orientation selectivity maps that emerge feature well-developed iso-orientation domains and fractures. During the last two phases of development the orientation preferences at some locations appear to rotate continuously through $\pm 180^{\circ}$ along circular paths and referred to as pinwheel-like patterns but without any corresponding point discontinuities in the orientation gradient maps. The formation of these functional maps is driven by balanced excitatory and inhibitory currents that are established via synaptic plasticity based on spike timing for both excitatory and inhibitory synapses. The stability and maintenance of the formed maps with continuous synaptic plasticity is enabled by homeostasis caused by inhibitory plasticity. However, a prolonged exposure to repeated stimuli does alter the formed maps over time due to plasticity. The results from this study suggest that continuous synaptic plasticity in both excitatory neurons and interneurons could play a critical role in the formation, stability, and maintenance of functional maps in the cortex.

Keywords: spiking networks, STDP, learning, functional maps, orientation selectivity, ocular dominance, stability

\section{INTRODUCTION}

The spatial and temporal properties of a distributed pattern of neural activity in V1 with differentially tuned responses of individual neurons to features of visual space such as orientation, spatial frequency, and direction of motion were first recognized by Hubel and Wiesel $(1962,1963,1968,2005)$. Using microelectrodes and neuroanatomical tracers, they established that the neural activity of a population of neurons with such differentiation represented functional maps. This seminal work has inspired a large body of subsequent research to understand the properties of these functional maps and its relation to cortical function as well as to understand the mechanisms by which these maps are formed during development (Blakemore and Cooper, 1970; Miller, 1996; Rao et al., 1997; Buonamano and Merzenich, 1998; Miller et al., 1999; Yuste and Sur, 1999; Basole et al., 2003, 2006; Hensch, 2005; Schummers et al., 2005; Yu et al., 2005; Shapley et al., 2007; White and Fitzpatrick, 2007; Huberman et al., 2008; Xing et al., 2011).

Two types of functional maps have been particularly well explored. The first map called the ocular dominance maps, or ODM, is based on interactions between axons of the neurons in the lateral geniculate nucleus (LGN) and neurons in layer 4 of V1. Here clusters of thalamocortical axon terminals that serve the left or right eye are organized in layer 4 via the topological relations established in the LGN to form ODMs. The second functional map called the orientation selectivity map, or OSM, is a map of orientation preference that is elaborated with a high degree of selectivity in V1 but not in the LGN. A key mechanism implicated in the formation of these functional maps during development is activity-dependent plasticity (Purves and Lichtman, 1985; Katz and Shatz, 1996; Ruthazer and Stryker, 1996; Crair et al., 1998; Crowley and Katz, 1999).

A number of in vitro experimental studies (Levy and Steward, 1983; Magee and Johnston, 1997; Markram et al., 1997; Bi and Poo, 1998; Debbane et al., 1998; Caporale and Dan, 2008) suggest that repeated pairing of pre- and postsynaptic activity in the form of action potentials, or spikes, can lead to long-term changes in synaptic efficacy. The sign and magnitude of the change in synaptic efficacy depends upon on the relative timing between the preand postsynaptic spikes and is known as spike-timing-dependent plasticity (STDP). STDP is now a well-established physiological mechanism of activity-driven synaptic regulation in vivo as well as observed in the Xenopus visual system (Mu and Poo, 2006; VislayMeltzer et al., 2006), the locust mushroom body (Cassenaer and Laurent, 2007), and rat visual (Meliza and Dan, 2006) and barrel (Jacob et al., 2007) cortex. STDP has also shown to have better explanatory power than more conventional Hebbian correlationbased plasticity at explaining both cortical reorganization in cat primary visual cortex (Young et al., 2007) and connectivity in locust olfactory system (Finelli et al., 2008). The STDP is a local learning rule that forces synapses to compete such that the spiking activity of a post-synaptic neuron becomes selective to a small 
subset of pre-synaptic input spikes. This feature was exploited in some spiking models to demonstrate map development (Bartsch and van Hemmen, 2000; Song and Abbott, 2001; Billings and van Rossum, 2009).

In the present study a spiking model is described that provides a plausible set of mechanisms based on STDP for the formation and maintenance of ODMs and OSMs. This developmental model simulates functional map formation during three phases: an early experience-independent phase, a late experienceindependent phase, and a subsequent refinement phase during which experience acts to shape map properties. There are other models that have also modeled developmental phases of functional maps in the visual cortex (Sirosh and Miikkulainen, 1995; Bauer et al., 2000; Swindale, 2000; Bednar and Miikkulainen, 2004; Yang et al., 2012). However, we present a spiking model that employs STDP as the basis to form and stabilize functional maps across the three phases of development. The resulting ODMs represent V1 neurons that show eye selectivity in response to two sets of monocular inputs from the LGN. The OSMs on the other hand feature pin wheel-like patterns to represent orientation preference in a smooth and continuous fashion. However, point discontinuities that appear at the center of these pin wheels in animal data (Maldonado et al., 1997) is not found in our model simulations. It also contains other forms of discontinuities such as fractures and breaks as will be defined in the next section.

\section{MATERIALS AND METHODS}

For the purpose of simplicity and clarity, the focus of this study will be on the dynamics of interaction between the neurons in LGN and layer 4 in V1. The model is developed in three phases where the first and second phases correspond to a pre-critical period while the third phase corresponds to a critical period (Hensch, 2005). In the early experience-independent phase, spontaneously generated neuronal activity in the cortex and LGN facilitates activity-dependent plasticity and formation of OSMs and ODMs (Wiesel and Hubel, 1974; Chapman et al., 1996; Crair et al., 1997; Ferster and Miller, 2000; Trachtenberg et al., 2000; Chiu and Weliky, 2001; Huberman et al., 2006). This is followed by a late-experience-independent phase where interactions between LGN and layer 4 are driven by the influence of retinal waves (Godfrey and Swindale, 2007) to enable the refinement of ODMs and OSMs (Crair et al., 1997; Crowley and Katz, 1999, 2002; Butts, 2002; Katz and Crowley, 2002; Huberman et al., 2006; Feller, 2009). In the final experience-dependent phase, neuronal activity is driven by natural visual stimuli from the environment that drives the maturation of the already formed ODMs and OSMs (Crair et al., 1998; Sengpiel et al., 1999; White et al., 2001; Coppola and White, 2004; Smith and Trachtenberg, 2007; White and Fitzpatrick, 2007). It should be noted that the model results are meant to show that the maturation of the formed OSMs and ODMs are driven by activity-dependent plasticity while qualitatively simulating some of the process constraints (such as the influence of retinal waves) during various stages of development. These maps have some similar qualitative properties as those found in animals but this does not imply actual adherence to the maps or process in any particular species.

\section{MODEL ARCHITECTURE}

The spiking model architecture in this study assumes that the initial structure of connections despite being random and local in nature is nevertheless present from the beginning. It is known that this initial formation of the map depends upon molecular gradients that serve as guides for axons to topologically appropriate portions of the map (Yuste and Sur, 1999; Crowley and Katz, 2002; Hensch, 2005; Taha and Stryker, 2005; Huberman et al., 2006; White and Fitzpatrick, 2007). The model also assumes that the neurons are mature unlike in reality where neurons are immature during very early stages of development and are characterized by a high concentration of $\mathrm{Cl}^{-}$ions as a result of which all neurons are depolarizing (Hensch, 2005).

The model is designed to address the thalamocortical circuit where thalamic afferents from the LGN activate the principal cells of layer 4 of V1 via geniculocortical synapses (Antonini and Stryker, 1978; Ursey et al., 1999; Yuste and Sur, 1999; Bartsch and van Hemmen, 2000). For convenience, the principal cells within layer 4 will be referred to as $E$ neurons while all the inhibitory interneurons will be referred to as $I$ neurons throughout the article. The $E$ neurons are connected to other local $E$ neurons and $I$ neurons to form a dense local recurrent network. In our model, the $E$ and $I$ neurons will make up two sub-layers within layer 4 . Similarly the LGN in the full model architecture (Figure 1) is also modeled as an Excitatory-Inhibitory $(E-I)$ network. The $E-I$ network model is a commonly used design to simulate models of thalamocortical areas (Binzegger et al., 2004; Kremkow et al., 2007; Kumar et al., 2008). The synapses in the model are plastic throughout all phases of development (Hensch, 2005) and the self-organization process refers to the change in the synaptic conductance during development.

In general, neurons in the cerebral cortex are densely connected to neurons close to it and sparsely connected to neurons far away from it (Schummers et al., 2004; Song et al., 2005; Perin et al., 2011; Voges and Perrinet, 2012). In particular, models of cortical function often assume that cortical circuitry acts in a center-surround fashion, whereas separated pairs of cells have a mutually suppressive influence. Further, to make the enhanced cortical patterns congruent with the sensory representation of the system, the cortical interactions must depend on the functional distance between the cells, determined by the features coded by them. This functional circuitry, known as "Mexican hat" organization, has been adopted in network models of orientation selectivity (Somers et al., 1995; Kang et al., 2003). In our model, we make a similar assumption where the Gaussian distribution of synaptic connections for the excitatory or glutamatergic synapses is dense and narrow in its spatial extent (Figure 2A) compared to the inhibitory or GABAergic synapses which are more broadly distributed.

The $E$ neurons in layer 4 (Figure 2B) are connected to its neighboring $E$ neurons and $I$ neurons in $I$ layer of layer 4 . Similarly, $I$ neurons in layer 4 are connected to its neighboring $I$ neurons as well as the $E$ neurons in layer 4 . Thus there are four types of synapses depending on the pre- and post-synaptic neuron: $E \rightarrow E, E \rightarrow I, I \rightarrow E$, and $I \rightarrow I$. The first two types of synapses are excitatory in nature and obey E-STDP rule while the last two synapses are inhibitory in nature and obey the I-STDP 


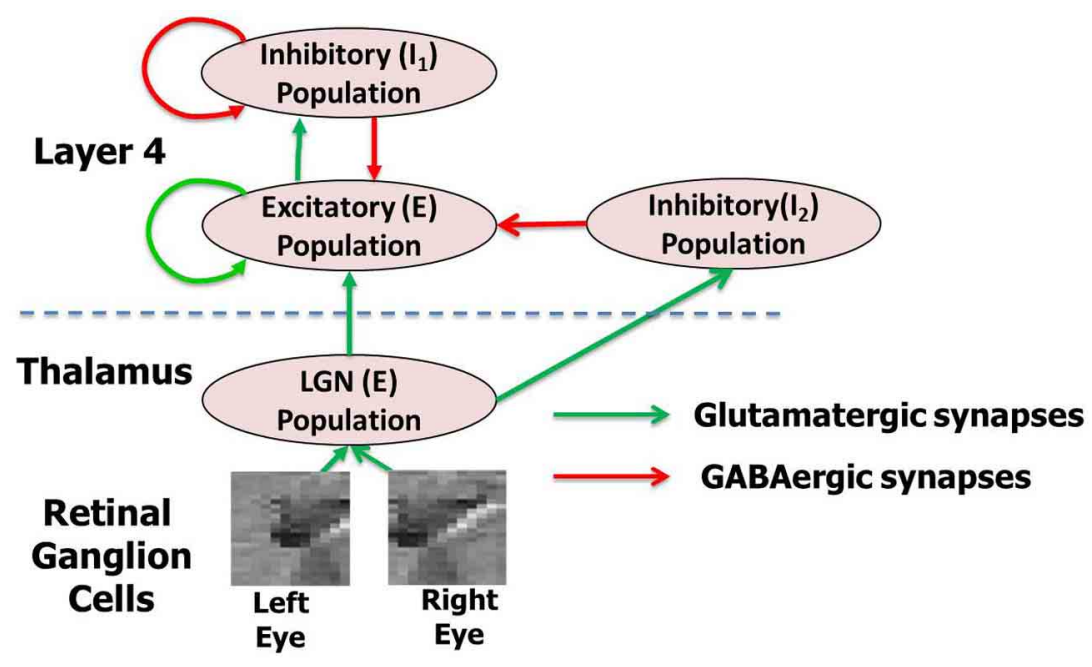

FIGURE 1 | The complete network model (A) with thalamocortical circuit where thalamic afferents from the LGN activate the principal cells of layer $\mathbf{4}$ of $\mathbf{V} \mathbf{1}$ via geniculocortical synapses. The layer 4 and LGN are both modeled as an $E-/$ network. There are two inhibitory populations in layer 4: the feedback inhibitory population $l_{1}$, which does not receive any inputs from LGN but only from the $E$ neurons of layer 4 and the feedforward inhibitory population $I_{2}$, which does. The LGN receives spikes from retinal ganglion cells (RGC). The LGN and layer 4 neurons in the model are separated by the dashed line in the figure. This complete model is used for simulating the experience-dependent phase of development. For each network layer, 60\% of randomly chosen neurons are injected with background noise in form of currents $\left(l_{\text {inj }}\right)$ for $30 \mathrm{~ms}$. A new of set of $60 \%$ randomly chosen neurons at all layers are selected again after that and are injected with background noise. This process is repeated throughout all three phases of development. For simulating early experience-independent phase (Phase 1) the complete network is purely driven by the background noise at both LGN and layer 4 . For simulating late experience-independent phase (Phase 2) the LGN is activated by spikes due to retinal waves from RGC. rule for plasticity (Woodin et al., 2003; Caporale and Dan, 2008; Hartmann et al., 2008). The synaptic connections are initialized with random synaptic strengths and obey the connection density as prescribed by the Gaussian distribution shown in Figure 2A for all four types of synapses. An example of a $10 \times 10$ set of $E$ neurons in layer 4 with $E \rightarrow E$ synaptic connectivity is provided in Figure 2D. Without loss of generality, the $E$ and $I$ layers in layer 4 in all our simulations will consist of a 2 -D sheet of $128 \times 128$ neurons. The LGN in the full model (Figure 1) is also composed of an $E-I$ network similar to layer 4 . The $E$ and $I$ layers within the LGN consist of $48 \times 48$ neurons and there are two such $E-I$ networks in the LGN corresponding to each eye. Each LGN $E$ neuron from both the eyes makes sparse and random synaptic connections to $E$ neurons in layer 4 (Figure 2C). In addition to these LGN $\rightarrow E$ synaptic connections, the LGN neurons are also connected to a feedforward inhibitory population of neurons which provide feedforward inhibition to the same $E$ neighborhood in layer 4. Feedforward inhibition is known to play a role in input normalization and expansion of cortical dynamic range (Pouille et al., 2009). The ultimate distribution of synaptic strengths in model synapses is dictated by E-STDP and I-STDP during the developmental process.

The spiking model simulations were performed using the HRLSim (Minkovich et al., under revision) which is a multiple graphical processing unit (GPU) based spiking simulator in $\mathrm{C}^{++}$. This simulator is an extension of a single GPU developed previously (Nageswaran et al., 2009) and uses an MPI interface and other optimizations to enable scalable and real-time simulation of large scale spiking neural networks. The computations to estimate the ODM and OSM were performed in MATLAB. The details of the neuronal and synaptic mechanisms and the performance metrics and measures used for the various experiments are now provided.

\section{NEURON MODEL}

In this study the leaky integrate and fire neuron model (Vogels et al., 2005) is used where each neuron receives multiple input current signals and the dynamics of its membrane potential $V$ can be determined as:

$\tau_{m} \frac{d V}{d t}=\left(V_{\text {rest }}-V\right)+\sum w(t)\left(E_{\text {ex }}-V\right)-\sum z(t)\left(E_{m}-V\right)+I_{\text {inj }}$

when $V$ reaches a threshold voltage $V_{T}$, the neuron fires a spike, and $V$ is reset to $V_{\text {reset }}$. This basic model provides several control variables for the membrane voltage including synaptic conductance $w$ (excitatory) and $z$ (inhibitory), membrane time constant $\tau_{m}$, reversal potentials $E_{\mathrm{ex}}$ and $E_{\mathrm{in}}$, and resting voltage $V_{\text {rest }}$. The parameter $I_{\text {inj }}$ represents the current injected into the neuron. Synaptic inputs to the neuron are modeled as conductance changes where a single pre-synaptic spike at time $t$ generates a synaptic conductance for excitatory and inhibitory synapses as follows:

$$
\begin{aligned}
& w(t)=w e^{\frac{-t}{\tau_{\mathrm{AMPA}}}} \\
& z(t)=z e^{\frac{-t}{\tau_{\mathrm{GABA}}}}
\end{aligned}
$$

where the time constants $\tau_{\mathrm{AMPA}}$ and $\tau_{\mathrm{GABA}}$ are used to model the kinetics of AMPA and GABA receptors. The value of the excitatory and inhibitory synaptic conductance $w$ and $z$ in Equation (1) 
A

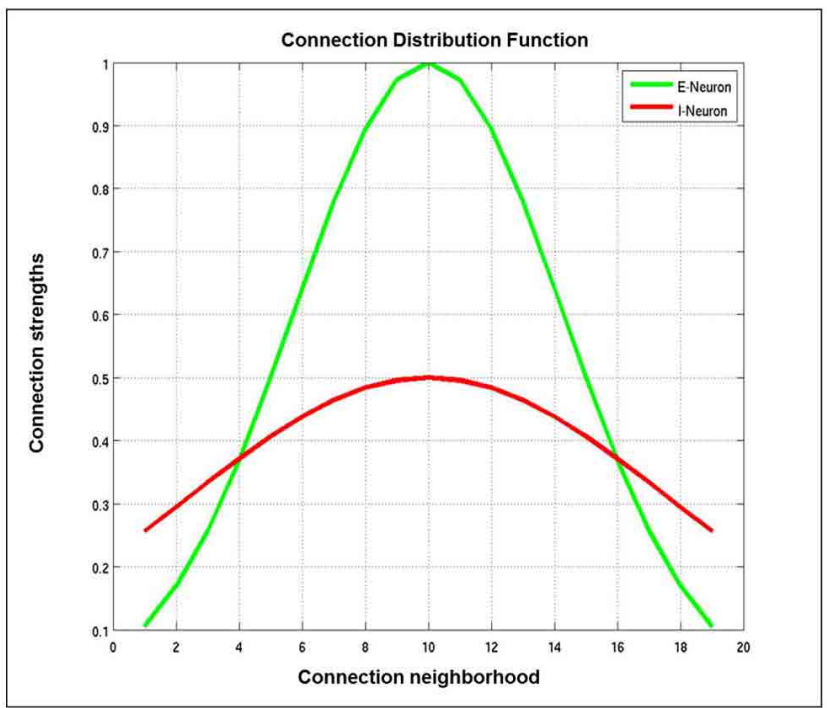

C

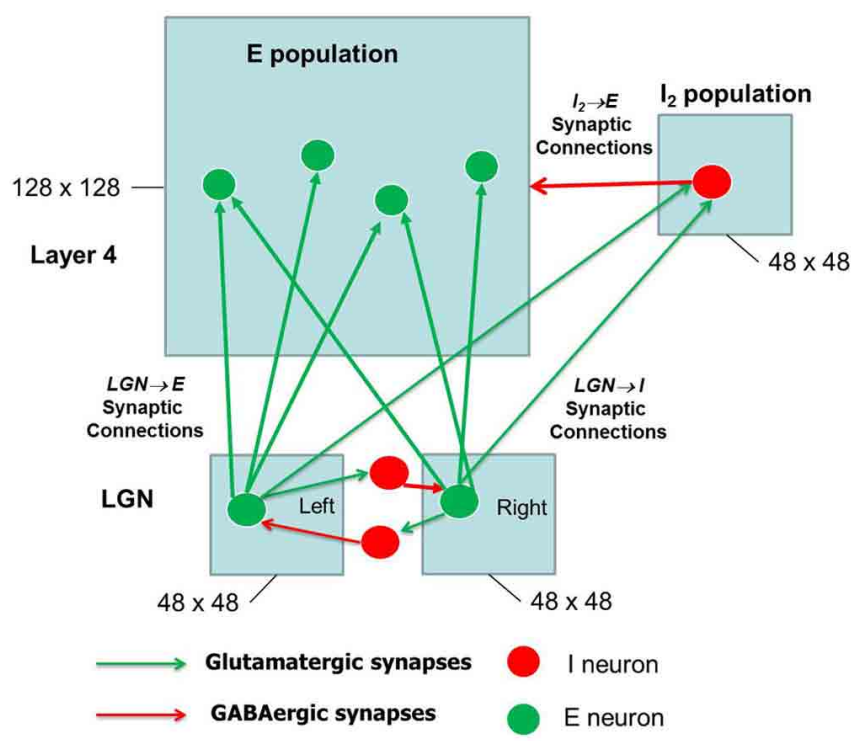

B

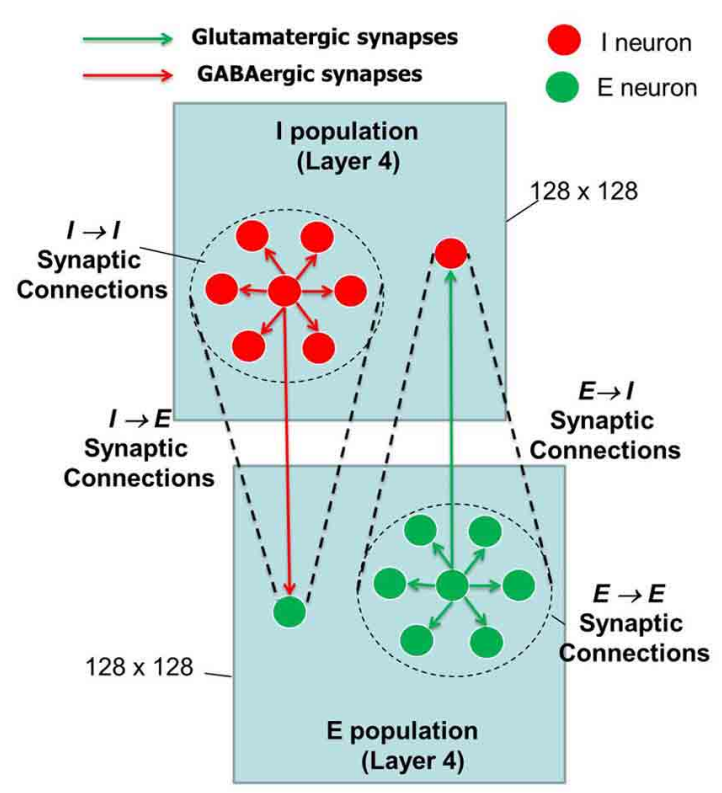

D

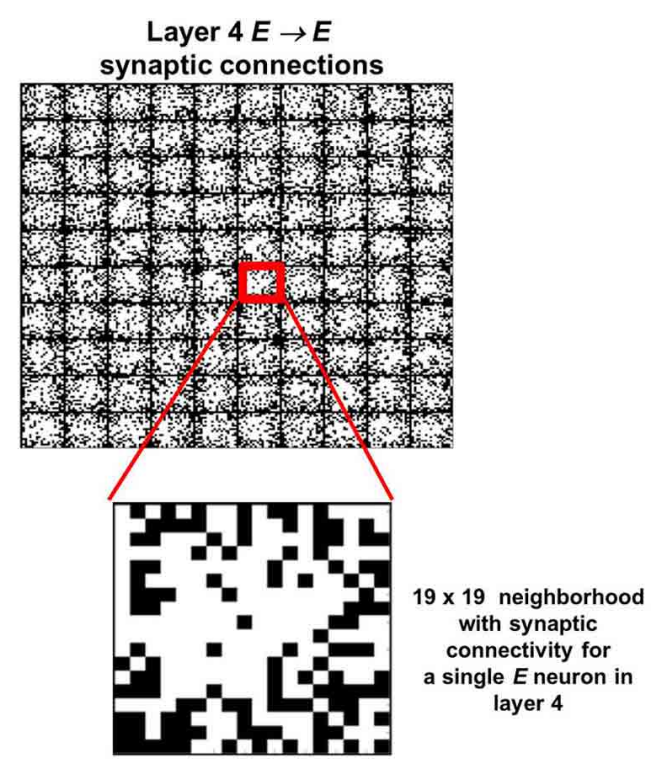

FIGURE 2 | The synaptic connectivity density distribution for the network. (A) From any $E$-neuron to any other neuron ( $E$ or $/$ ) in shown in green and from any I-neuron to any other neuron ( $E$ or $I)$ is shown in red. (B) There are four types of synapses depending on the pre- and post-synaptic neuron: $E \rightarrow E, E \rightarrow I, I \rightarrow E$, and $I \rightarrow I$. It should be noted that the network has periodic boundary conditions such that topmost and bottommost neurons are regarded as neighbors, as are the leftmost and rightmost columns within each layer. The neighborhood around each $E$ or I neuron is shown as a dotted circle. (C) The LGN network is also an E-I network as shown here with mutually inhibiting connections between neurons that receive inputs from the RGCs (not shown) from the left and right eye. Each LGN neuron from both eyes project to a neuron and its neighborhood in layer 4. For convenience, only one such projection is shown here. The LGN network $(2 \times 48 \times 48)$ is smaller than the layer 4 network $(128 \times 128)$. In addition, the LGN inputs from the left and right eye populations project to the 12 population in layer 4 which consists of $48 \times 48$ inhibitory neurons as well. (D) The synaptic connections for a set of $10 \times 10 E$ neurons in layer 4 are shown here. The red square shows a single $E$ neuron with a $19 \times 19$ neighborhood. The white pixels within each such square indicate synaptic connections with maximum synaptic strength while black pixels indicate synaptic connections with zero synaptic strength. A closer look at the $19 \times 19$ neighborhood for one of $E$-neuron shows the initial strengths of synapses from the $E$-neuron in the center to its neighboring $E$ neurons. These synaptic strengths are randomly distributed. 
Table 1 | Neuron parameters.

\begin{tabular}{lc}
\hline Parameter & Value \\
\hline$\tau_{m}$ & $20 \mathrm{~ms}$ \\
$E_{\text {Ex }}$ & $0.0 \mathrm{mV}$ \\
$E_{\text {inh }}$ & $0.0 \mathrm{mV}$ \\
$V_{\text {reset }}$ & $-60 \mathrm{mV}$ \\
$V_{\text {rest }}$ & $-74 \mathrm{mV}$ \\
$V_{T}$ & $-54 \mathrm{mV}$ \\
$\tau_{\text {AMPA }}$ & $10 \mathrm{~ms}$ \\
$\tau_{\text {GABA }}$ & $50 \mathrm{~ms}$ \\
\hline
\end{tabular}

is determined by STDP. Table 1 provides a list of values for all the constants used to simulate the neuron model. During model simulations, the continuous membrane equation is updated using Euler integration with a time step of $1 \mathrm{~ms}$.

\section{EXCITATORY STDP}

The E-STDP function modulates the excitatory synaptic conductance $w$ based on the timing difference $\left(t_{\text {pre }}-t_{\text {post }}\right)$, or $\Delta t$, between the spike times of pre- and post-synaptic neuron (Figure 3A). The control parameters $\left(A^{+}, A^{-}, \tau^{+}, \tau^{-}\right)$can be used to modify the amount of potentiation and depression (Table 2). The synaptic conductance is computed as:

$$
w_{\text {new }}=w_{\text {old }}+\Delta w
$$

where

$$
\Delta w=g_{\max } \times F(\Delta t)
$$

and

$$
F(\Delta t)= \begin{cases}A_{+} \times \exp \left(\frac{\Delta t}{\tau_{+}}\right), & \text {if } \Delta t<0 \\ -A_{-} \times \exp \left(\frac{\Delta t}{\tau_{-}}\right), & \text {if } \Delta t \geq 0\end{cases}
$$

If $w_{\text {new }}>g_{\max }^{E}$, then $w_{\max }=g_{\max }^{E}$. On the other hand if $w_{\text {new }}<0$, then $w_{\text {new }}=0$. The factor $\beta=\left|A^{-} \tau^{-}\right| /\left|A^{+} \tau^{+}\right|$controls the relative amounts of depression to potentiation during learning.

\section{INHIBITORY STDP}

The I-STDP function modulates the inhibitory synaptic conductance $z$ based on the timing difference $\Delta t$ between the spike times of pre- and post-synaptic neuron (Figure 3B). The synaptic conductance is computed as:

$$
z_{\text {new }}=z_{\text {old }}+\Delta z
$$

The change $\Delta z=B^{+}$is more inhibitory when $-\lambda \leq \Delta t \leq \lambda$. The change $\Delta z=B^{-}$is less inhibitory when $-\lambda-\bar{\delta} \leq \Delta t<$ $-\lambda$ or $\lambda<\Delta t \leq \lambda+\delta$. There is no change in inhibition if either $\Delta t \leq-\lambda-\delta$ or $\Delta t \geq \lambda+\delta$. If $z_{\text {new }}<0$ then $z_{\text {new }}=0$. On the other hand, if $z_{\text {new }}>g_{\text {max }}^{I}$ then $z_{\text {new }}=g_{\text {max }}^{I}$. The control parameters $\left(\mathrm{B}^{+}, \mathrm{B}^{-}, \lambda\right.$, and $\delta$ ) (see Table 2 ) can be used to vary the relative amounts of potentiation and depression during learning.

\section{MODEL SYNAPTIC CONNECTIVITY}

The synaptic connectivity in layer 4 and LGN is initialized using a Gaussian density function as:

$$
p(x, y)=\alpha \times e^{\frac{-\left[\left(x-x_{0}\right)^{2}+\left(y-y_{0}\right)^{2}\right]}{2 \times \sigma}}
$$

The point $\left(x_{0}, y_{0}\right)$ represents the center neuron and $(x, y)$ represents the position of neurons within its neighborhood. The constants $\alpha$ and $\sigma$ (Table 3 ) control the maximal distribution probability and distribution variation.

\section{BACKGROUND SIGNAL AND NOISE}

A random voltage injection is used to mimic cortical input spikes generated from brain regions (or background) as well as noise. The injected voltage $V_{\text {inj }}(t)$ is modeled as:

$$
V_{\text {inj }}(t)=A u(r), \quad t \in\left[t_{1}, t_{1}+\Delta t\right]
$$

with

$$
u(r)= \begin{cases}1 & r \geq \mu \\ 0 & \text { otherwise }\end{cases}
$$

The constant $A$ determines the amplitude of voltage injection. The variable $r$ is a random variable uniformly distributed in the range $(0,1.0)$. The parameter $\Delta t$ (Table 3$)$ is the voltage injection duration while the parameter $\mu$ represents the percentage of neurons that receive voltage injection randomly. Thus, the injection time duration $\Delta t$ is used to set an injection frequency. In our model, before the eyes open, the spiking activity of neurons is driven primarily by random current injection $I_{\text {inj }}$ (in Equation 1) by multiplying $V_{\text {inj }}(t)$ into currents using a fixed synaptic conductance constant of $0.00125 \mathrm{nS}$.

\section{RETINAL WAVE MODEL}

Before the onset of stimulus driven activity, which helps refine neural organization in later developmental stages, neural circuits generate spontaneous patterns of activity which guide early development (Katz and Shatz, 1996). In the retina, spontaneous activity takes the form of coordinated bursts of spikes in the neighboring retinal ganglion cells (RGC) that slowly spread across the retina. They can initiate at any retinal location and cover the entire retina in minutes (Schiller, 1992; Butts, 2002; Godfrey and Swindale, 2007; Feller, 2009). The retinal waves were generated in our model as follows. The retina is assumed to have $48 \times 48$ neurons or RGCs. For each eye, a randomly chosen set of $N$ RGCs are selected for retinal wave initiation. For each initiation site, a direction $d$ is selected for wave propagation from a set of eight possible directions (i. e., $0^{\circ}, 45^{\circ}, 90^{\circ}, 135^{\circ}, 180^{\circ}$, $225^{\circ}, 270^{\circ}$, and $315^{\circ}$ ). At each initiation site, RGCs within a neighborhood of size $10 \times 10$ are then activated at the first time step $(t=1 \mathrm{~ms})$. The strength of the stimulation is varied in a Gaussian fashion where neighbors at closer distance are stimulated more strongly than neighbors that are further apart. In the next time step ( $t=2 \mathrm{~ms}$ ) the next RGC to be stimulated is selected after moving by two steps (i.e., velocity is 2 steps/ms) in the direction $d$. The newly stimulated RGC is used to activate 
A

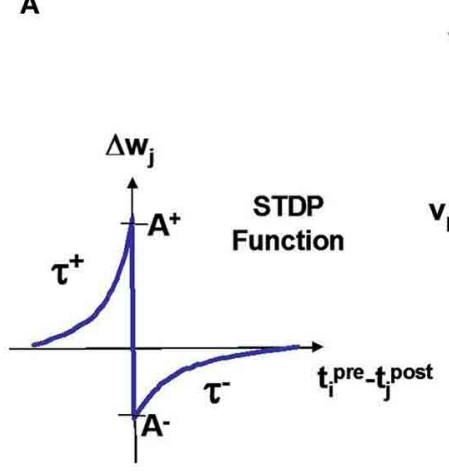
Depression

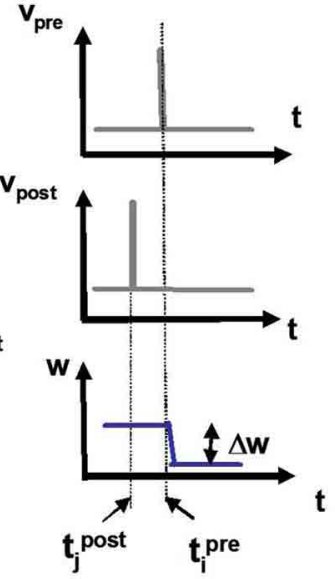

B

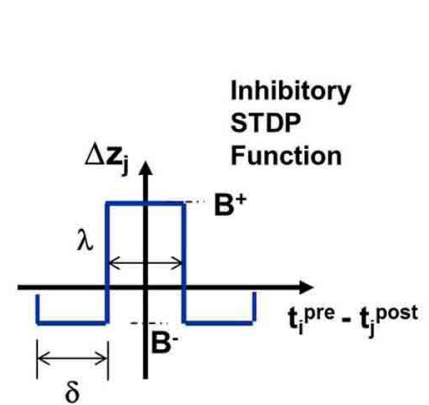

$\delta$
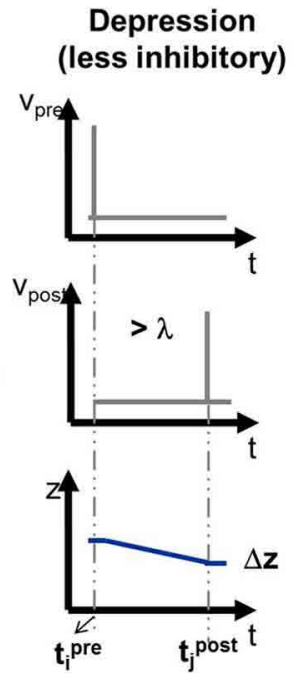

Potentiation

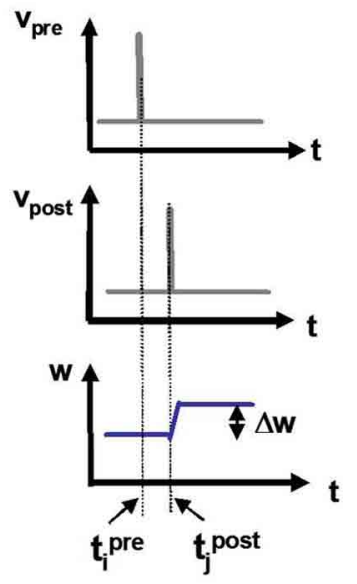

Potentiation

(more inhibitory)

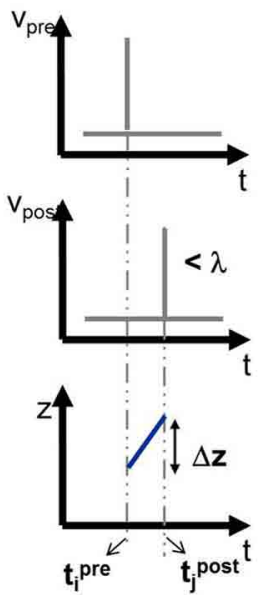

FIGURE 3 | (A) The E-STDP function modulates the excitatory synaptic conductance $w$ based on the timing difference $\left(t_{i}^{\text {pre }}-t_{j}^{\text {post }}\right)$ between the action potentials of pre-synaptic neuron $i$ and post-synaptic neuron $j$. The control parameters $\left(A^{+}, A^{-}, \tau^{+}\right.$, and $\left.\tau^{-}\right)$can be used to modify the amount of potentiation and depression (see "Materials and

Methods"). (B) The I-STDP function modulates the inhibitory synaptic conductance $z$ based on the timing difference $\left(t_{i}^{\text {pre }}-t_{j}^{\text {post }}\right)$ between the action potentials of pre-synaptic neuron $i$ and post-synaptic neuron $j$ (see "Materials and Methods"). If the timing difference is $>\lambda$, then the synapses become less inhibitory and the change itself is of a smaller magnitude while it is the opposite case when the timing difference is $<\lambda$
RGCs within a $10 \times 10$ neighborhood and the process is continued. The total duration for each retinal wave for each eye is set to $10 \mathrm{~ms}$. The activity of the RGCs is converted into spike trains as described below. The process is continued to generate several retinal waves during the late activity-independent developmental phase.

\section{SPIKE ENCODING OF RETINAL WAVES AND NATURAL IMAGES}

The input visual images are in the form of either retinal waves or other natural images. For natural images, we use the Caltech 101 image dataset (Fei et al., 2006). A total of two thousand $48 \times 48$ images were cropped from the database as follows. A subset of images was selected from the database such that the central portion of the image had some texture and contrast in them.
These original images of size $320 \times 200$ were down-sampled to $128 \times 128$ images and then a $48 \times 48$ portion from the center of these images (see Figure 11) was extracted. These $48 \times 48$ images were provided as RGC inputs to the LGN after spike encoding the images as described below. While the Caltech 101 dataset was developed for object recognition purposes, the statistics of the images extracted is still representative of natural images in general.

We created stereo pairs from every image in the dataset by shifting the right eye image by a maximum of 10 pixels to the left or right and 10 pixels to the top or bottom with respect to the left image. This simulates disparity in the two images. In addition to disparities, we also scale the right image by a small scaling factor between 1 and 1.05 to simulate small perspective changes. Such 
Table 2 | STDP parameters.

\begin{tabular}{lll}
\hline STDP & Parameter & Value \\
\hline E-STDP & $g_{\max }^{E}$ & \\
& $\mathrm{~A}^{+}$ & $0.035 \mathrm{nS}$ \\
& $\beta$ & $0.0 \mathrm{mV}$ \\
& $\tau^{+}$ & $1.02 \mathrm{nS}$ \\
$\tau^{-}$ & $20 \mathrm{~ms}$ \\
I-STDP & $20 \mathrm{~ms}$ \\
& $g_{\max }^{\prime}$ & \\
& $\mathrm{B}^{+}$ & $0.0035 \mathrm{nS}$ \\
& $\mathrm{B}^{-}$ & $0.045 \mathrm{nS}$ \\
$\lambda$ & $0.025 \mathrm{nS}$ \\
& $\delta$ & $12 \mathrm{~ms}$ \\
& $40 \mathrm{~ms}$ \\
\hline
\end{tabular}

Table 3 | Other model parameters.

\begin{tabular}{lll}
\hline STDP & Parameter & Value \\
\hline SYNAPTIC CONNECTIVITY & \\
& $\sigma_{E}$ & 1.0 \\
& $\sigma_{l}$ & 0.5 \\
& $\alpha_{E}$ & 2.5 \\
& $\alpha_{l}$ & 3.5 \\
$\tau^{-}$ & $20 \mathrm{~ms}$ \\
BACKGROUND AND NOISE & \\
& $\mathrm{A}$ & 1.07 \\
& $\Delta t$ & $30 \mathrm{~ms}$ \\
& $\mu$ & 0.65 \\
& $t_{1}$ & $0 \mathrm{~s}$ \\
& $t_{2}$ & $6000 \mathrm{~s}$ \\
OSM COMPUTATION & & \\
& $\phi$ & $0^{\circ}, 45^{\circ}, 90^{\circ}, 135^{\circ}$ \\
& $\sigma_{a}$ & 2.5 \\
$\sigma_{b}$ & 5.7 \\
$\Upsilon$ & {$[-8.0,8.0]$} \\
\hline
\end{tabular}

affine transforms are commonly used in computer vision (Zhang and $\mathrm{Xu}, 1998)$.

The images are converted into spike sequences by an encoding process, and the sequences serve as input to neurons in the LGN layer. The encoding process is generated based on Poisson statistics where the Poisson distribution is used to generate the interspike interval (ISI) for each pixel. The mean value of pixel intensities in the image serves as the mean value of the Poisson distribution. The Poisson distribution is given as:

$$
P(x)=\frac{\lambda^{x} e^{-\lambda}}{x !}
$$

where $\lambda$ is the mean value of pixel intensities and $x$ is the ISI. To generate the spikes for a given pixel at a given time, we randomly select a probability value $P(x)$ at each time step and then compute $x$ using Equation (11). This sets the time step at which the next spike is generated as part of the encoding process.

\section{RECURRENT CORTICAL MAP COMPUTATION}

The recurrent cortical map (RCM) computation is based on the synaptic conductances between $E \rightarrow E$ neurons in layer 4 . The purpose of RCM computation is to identify evolution of local synaptic connectivity within the $E$ sub-layer of layer 4 during the various phases of development. The RCM is estimated by using a Gaussian bar function as an orientation template (Bartsch and van Hemmen, 2000) to search for the best orientation match within its neighborhood. The $E$ neurons are color coded based on the best match score. The resulting image of the color coded $E$ neurons constitutes a RCM.

Assuming that an $E$ neuron is located at $p$, the Gaussian bar function is given by:

$$
\begin{aligned}
G_{x y}(\phi, p)= & \exp \left\{\frac{-[x \cos \phi+y \sin \phi-\gamma]^{2}}{2 \times \sigma_{a}^{2}}\right\} \\
& \times \exp \left\{\frac{-[y \cos \phi-x \sin \phi]^{2}}{2 \times \sigma_{b}^{2}}\right\}-G_{0}(\phi, p)
\end{aligned}
$$

where the variables $x$ and $y$ varies within $E$-neuron's locally connected neighborhood and $\sum_{x y} G_{x y}(\phi, \gamma)=0$. For each of the four orientations, $\phi \in\left\{0^{\circ}, 45^{\circ}, 90^{\circ}, 135^{\circ}\right\}$, the parameter $\gamma$ is varied to determine the maximal orientation match as:

$$
R(\phi)=\max _{p}\left[\sum_{x y} w(x, y) G_{x y}(\phi, \gamma)\right]
$$

With the maximal orientation match, a direction vector $\vec{d}(\phi)=$ $(R(\phi), 2 \phi)$ is constructed for each orientation. Then, the four direction vectors of the four orientations are combined into a final direction vector as:

$$
\vec{S}=\left(R_{s}, \phi_{s}\right)=\vec{d}\left(0^{\circ}\right)+\vec{d}\left(45^{\circ}\right)+\vec{d}\left(90^{\circ}\right)+\vec{d}\left(135^{\circ}\right)
$$

The orientation of synaptic weights for the E-neuron is determined by

$$
\phi_{\text {or }}=\phi_{s} / 2
$$

The RCM is obtained by color coding each $E$ neuron in layer 4 using $\phi_{\text {or }}$. The RCMs does not reflect orientation selectivity of OSMs since they are not measured based on inputs from the LGN and only show anisotropies in the local pattern of connectivity within layer 4.

\section{ODM COMPUTATION}

The ODM was computed using the following procedure. Each $E$ neuron in layer 4 receives inputs from $E$ neurons in the LGN. The initial synaptic conductance values for all input synapses from LGN are set to the same value. Thus, the $E$ neurons initially start off by responding equally to inputs from both the eyes and are thus labeled as binocular. These geniculocortical synapses are tuned using STDP during the three phases based on random activity, retinal waves and with natural stimuli. The number of afferent synapses into an $E$ neuron of layer 4 after each phase of development is tracked from each eye and the $E$ neuron is 
assigned a membership to one of the two eyes based on the eye for which the sum of synaptic conductances from LGN $\rightarrow E$ is greater. It should be noted that this eye selectivity naturally emerges without the need for setting any arbitrary thresholds due to two factors. The first factor is due to mutual inhibition between the LGN neurons corresponding to the left and right eye (see Figure 2C). I-STDP in these inhibitory synapses forces the LGN neurons to compete and this competition affects the ability of $E$ neurons in LGN to influence the $E$ neurons in layer 4 . The second factor is that E-STDP in the synapses from LGN $\rightarrow E$ pathway also creates a competition between synapses from the two eyes (Miller et al., 1989). These two competitive factors combine to break the symmetry in the geniculocortical synapses from the two eyes by increasing the synaptic conductances for inputs from the eye that attains a positive bias and vice versa.

Over time, if there is some spatial structure in the inputs (as it happens after Phase 1), the synaptic conductances in the LGN $\rightarrow E$ pathway become more separated in magnitude based on the eye due to aforementioned competition until the difference becomes stable. This causes the inputs from one eye in the LGN to eventually dominate the response of $E$ neurons in layer 4 . The reason for the appearance of contiguous patches of eye preference is due to the stable formation of the RCM. This map indicates local structure in the lateral excitation of neighboring $E$ neurons (see Figure 2B) in layer 4 to similar stimuli. Thus, as a given $E$ neuron develops a preference for one eye due to bottom-up competition as described above, the neighboring $E$ neurons in layer 4 also tend to receive excitation from this $E$ neuron and thus can influence their preference as well over time such that a well-developed and stable ODM emerges.

In order to measure the emergence of eye selectivity during various phases of development, we compute the sum of synaptic conductances for the geniculocortical synapses from the left and right eye separately for each $E$ neuron. We then compute the average and standard deviation of the difference between the two sums for all the E neurons in layer 4. If the ODMs are truly mature, then this average should be substantial in terms of the total dynamic range of the synaptic conductances while the standard deviation must be very small. Furthermore, these values must also stabilize over time indicating that the ODMs are both mature and stable. It should be noted that ODM maps generated in all phases are smoothed using a median filter of size $3 \times 3$ to remove small speckle noise due to some neurons developing opposite eye preference on occasion in a neighborhood of $E$ neurons that have the same eye preference.

\section{OSM COMPUTATION}

For clarity, we follow the definition of orientation preference and orientation selectivity provided in Blasdel (1992). Orientation preference of an $E$ neuron in layer 4 is the orientation that yields the strongest response while orientation selectivity is the rate at which responses fall to zero with increasing displacement from the preferred orientation. The orientation selectivity of $E$ neurons in layer 4 cannot be computed using RCMs since that computation does not involve input stimuli from LGN to the $E$ neurons of layer 4. In order to derive OSMs, we stimulate the LGN at a given location $p$ within it (on both eyes) using an oriented rectangular bar with a length of 15 pixels and a width of 5 pixels. At the end of each phase of development, the oriented bar in a given orientation $\alpha$ is moved bilaterally by different amounts (each amount being less than $<7$ pixels) during odd and even trials within the LGN layers and the resulting spikes from the stimuli (Equation 1) are used to stimulate the $E$ neurons that receive these spikes in layer 4 . The firing rate of spikes generated by each $E$ neuron in layer 4 in response to the LGN inputs is calculated by counting the number of spikes in a $10 \mathrm{~s}$ (or 10,000 steps) time window. The resulting spike activity provides a sense for which $E$ neurons respond more strongly to the input spikes from LGN for a given bar orientation. In other words, it provides an estimate of orientation selectivity of the $E$ neurons in layer 4 to that particular oriented bar in the LGN. This process is repeated for four different orientations of the bar (i. e., $\alpha=0^{\circ}, 45^{\circ}, 90^{\circ}$, and $135^{\circ}$ ).

Using the firing rates of $E$ neurons we then apply the algorithm described in Blasdel (1992) to compute OSM as follows. First, we compute the differential between positive and negative images corresponding to complimentary stimuli-for example, $\alpha=0^{\circ}$ and $\alpha=90^{\circ}$ would correspond to a positive and negative image. The negative image is subtracted from the positive and this reveals the change in the response to the stimulation due to the horizontal and vertical bar in the LGN. The differential image is then transformed at every location, in every image, into vectors displayed either as cosine and sine pairs, in Cartesian coordinates, and as magnitudes and angles in polar coordinates with angles corresponding to twice the positive stimulus orientation, and lengths corresponding to net intensities. The resulting vectors from the transformed images are added to reflect orientation-weighted contributions from each image. Since stimulus orientations are multiplied by two, contributions generated by similar orientations reinforce one another, while those generated by orthogonal orientations cancel. The polar coordinates (angles and magnitudes) that are computed from the summed image reflect the orientation preference and selectivity of the E neurons in layer 4 (Blasdel, 1992; Miikkulainen et al., 2005).

The orientation preferences are analyzed with respect to their gradient that measures the rate of change at every point in two dimensions. Following Blasdel, we compute the gradient in $x$ and $y$ and then convert the result to Polar coordinates. The magnitude of the gradient calculated from the polar coordinates indicates the steepest rate of change at any point irrespective of direction. The gradient magnitude is used to find discontinuities which appear as short lines or dots running across a region of continuous tone (Blasdel, 1992). These discontinuities can signify either a fracture if it appears as a short line between two contiguous regions with a gradient of $90^{\circ}$ or more, or signify pinwheel formation, if the gradient magnitude plot contains dots. This entire process described above was repeated after each developmental phase to assess the formation of OSM.

In order to get robust orientation gradients as well as orientation selectivity and preferences, the Blasdel approach averages the raw data from the summed Cartesian images (see Figure 13 in Blasdel, 1992). The purpose of this averaging process was to smooth out noise and improve the signal-to-noise ratio in these images. We smooth the summed $x$ and $y$ Cartesian images from our model using a median filter and then convert them into polar 
representation to obtain the orientation preference and selectivity of the $E$ neurons. In order to estimate the correct filter size for smoothing, we adopt an iterative convergence process as follows. We first compute the orientation gradient responses for the raw OSMs. The raw OSM data in our model has small patches of noisy neuron orientation preference responses amidst regions of uniform orientation preference. The orientation gradients computed with such noise yields spurious discontinuities in the form of extraneous dots and lines. To mitigate this, we gradually increase the filter size of the median filter and repeat the above process to compute the orientation gradients until there are no spurious discontinuities due to those noisy neurons. This process does not change the resulting orientation preference maps in any qualitative fashion but filters out the noisy neuron preferences. We find that a median filter of $9 \times 9$ was sufficient to avoid any orientation gradient artifacts due to noisy orientation preferences. This approach was used to compute orientation selectivity, orientation preference and orientation gradients as well as perform analysis on the resulting functional maps after each phase of development.

While the smoothing operation during OSM computation based on the Cartesian images (as described above) helps remove the salt and pepper noise in the orientation gradient maps to produce robust fractures, they also remove any point singularities as well. However, visual inspection of the OSMs shows that the orientation preferences rotate continuously through $\pm 180^{\circ}$ along circular paths. This feature is characteristic of a pinwheel (Ohki et al., 2006). A better model of how the signal is smoothed in animal experiments could possibly help mitigate this problem. At this point, since our methodology does not obtain precise point singularities in the orientation gradient maps, we call these formations pinwheel-like patterns.

\section{STABILITY ASSESSMENT}

In order to measure the stability of functional map or RCM, a similarity measure between the synaptic conductance maps that reflect that particular structure in one developmental phase is compared against the synaptic conductance maps in another developmental phase based on Kullback-Leibler (KL) distance (Kullback, 1987). For example, the stability of the RCMs is determined by the least change in the KL distance. Mathematically, let $h_{1}(w)$ and $h_{2}(w)$ represent the two synaptic conductance histograms for synaptic conductance maps from two different developmental phases, $W_{1}$ and $W_{2}$. By normalizing the histograms, the synaptic conductance distribution functions are obtained as:

$$
p_{i}(w)=\frac{h_{i}(w)}{\max \left[h_{i}(w)\right]}, \quad i=1,2 .
$$

The similarity between synaptic conductance maps is then computed as:

$$
\begin{aligned}
S\left(W_{1}, W_{2}\right)= & \frac{1}{2}\left[\sum_{w} p_{1}(w) \log \left(\frac{p_{1}(w)}{p_{2}(w)}\right)\right. \\
& \left.+\sum_{w} p_{2}(w) \log \left(\frac{p_{2}(w)}{p_{1}(w)}\right)\right]
\end{aligned}
$$

This KL measure provides a way to track changes in synaptic conductance during development and thus provides a measure of stability in the evolving functional maps or RCMs.

\section{RESULTS \\ PHASE 1: EARLY EXPERIENCE-INDEPENDENT OSM AND ODM FORMATION}

The first phase of development begins with an E-I network that models the local recurrent microcircuit in layer 4 (Figure 1). This network consists of a 2-D lattice of $128 \times 128$ neurons with a $19 \times 19$ local neighborhood of synaptic connections to other $E$ neurons (see Figures 2A,B). Neurons on the borders were assumed to have periodic boundary conditions such that topmost and bottommost neurons are regarded as neighbors, as are the leftmost and rightmost columns within each layer. The neurons exhibit spontaneous spiking activity (Chiu and Weliky, 2001; Huberman et al., 2006, 2008). This condition is simulated by injecting a constant small background current into a sub-population of both $E$ and $I$ neurons in layer 4 and LGN neurons of the network (see "Materials and Methods"). The conductance based synapses along with the recurrent connections within layer 4 ensures that the network is able to maintain this spontaneous activity (Kumar et al., 2008). The key aspect of the early experience-independent phase in our model is the absence of any retinal inputs (Figure $2 \mathbf{B}$ ).

As the neurons in the network begin to spike due to spontaneous activity, STDP alters the synaptic strengths $w$ and $z$ (Equations 6, 7) and thus the connectivity between various neurons. Using our initial network model architecture (see "Materials and Methods") combined with STDP in both excitatory and inhibitory synapses helps to achieve a good balance of excitation and inhibition. It is becoming more apparent that the ongoing balance of cortical excitation and inhibition plays a role in early development (Xing et al., 2011). The interesting aspect of our model is that inhibitory plasticity helps maintain this balance in the cortex that is qualitatively similar to observations in some recent experimental studies (Akerman et al., 2002)

This aspect of development was tested by performing three types of experiments. In the first experiment, both $w$ and $z$ synapses obeyed STDP and in this case the network is able to operate at much lower firing rates (average of $\sim 10 \mathrm{~Hz}$ ). As $w$ strengthens and create an imbalance in synaptic currents, $z$ get rapidly potentiated due to an order-independent I-STDP where inhibition increases irrespective of the order of occurrence of pre- and post-synaptic spikes (Caporale and Dan, 2008) for small timing differences between pre- and post-synaptic spikes (Equation 7). This results in a rapid compensatory increase in inhibitory currents into the neurons in a self-organized manner thus effectively preventing the neurons from exceeding $V_{T}$ more often (Vogels et al., 2011). This enables a good balance between excitation and inhibition and results in the emergence of RCM that have a locally smooth structure (Figure 4A).

In the second experiment all inhibitory synapses are turned off (i. e., $z=0$ ). It was observed that firing rate of the $E$ neurons was high on average $(\sim 100 \mathrm{~Hz})$ but there was no emergent structure (Figure 4B). A high firing rate of neurons due to lack of inhibition forces the synapses to compete at a faster rate due 


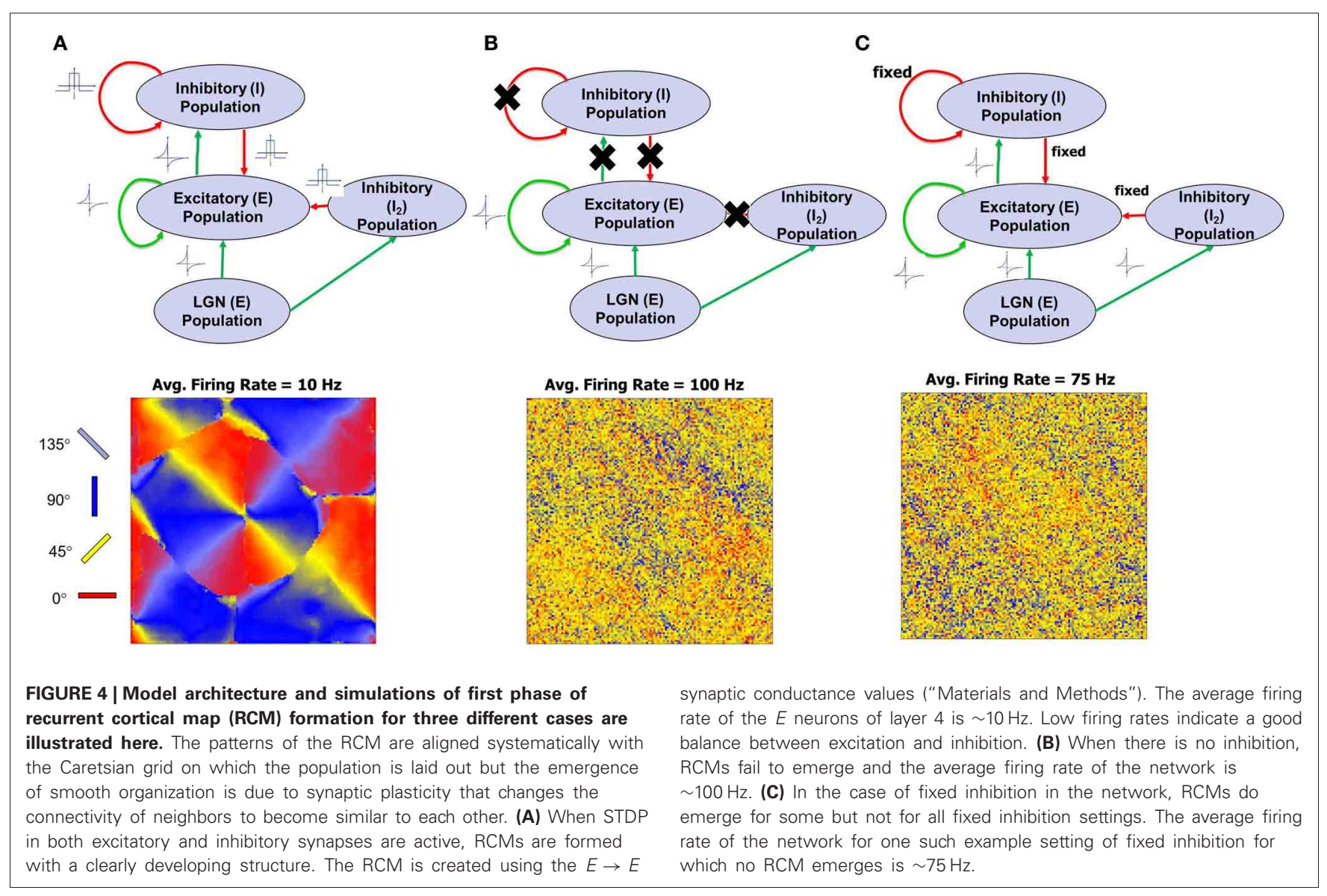

to the asymmetric E-STDP rule (Equation 6) that results in a rapid rise in synaptic conductances for some synapses and a rapid fall in synaptic conductances for most others (due to a bias toward depression-see "Materials and Methods"). These rapid changes in synaptic conductance appear to be detrimental to the emergence of structure.

In third experiment excitatory synapses obeyed the STDP rule while plasticity was turned off for all the inhibitory synapses. Instead the inhibitory synapses were fixed (i. e., $z=$ const) in synaptic strength. The exact setting for the fixed inhibitory synaptic conductance values was critical in order to see any emergence of structure. The firing rate was lower on average $(\sim 75 \mathrm{~Hz})$ and was not conducive for the emergence of structure in most cases (Figure 4C).

The emergence of RCM during the first phase of development is captured in Figure 5. The time taken for the emergence is just an artifact of the model parameter settings and reflects upon the fact that activity-dependent plasticity caused by STDP enables the emergence of RCMs (see "Materials and Methods"). The distribution of $E \rightarrow E$ synapse conductances (Figure 5A) shows smoothly varying structure in synaptic conductances within a neighborhood (Figure 5B). The distribution of synaptic conductances (Figure 5C) shows that the initial bimodal distribution becomes more separated and sparse in strong connections that is due to the competitive nature of STDP (Song and Abbott, 2001) and this competition between synapses that are pre-synaptic to neurons in layer 4 results in the modification of their synaptic connectivity and results in the emergence of RCMs.

In order to test the orientation selectivity of $E$ neurons in layer 4, the following experiment was performed (see "Materials and Methods"). The LGN neurons from both eyes were stimulated with oriented bar stimuli and the spiking activity of $E$ neurons in layer 4 were measured in time windows of $10 \mathrm{~ms}$ and then averaged to compute their firing rates. The goal was to observe if there was preferential firing of V1 neurons to certain orientation of the bar stimuli presented in the LGN. A strong selectivity response feature required the adaptation of both the geniculocortical (LGN $\rightarrow E$ ) and cortico-cortical $(E \rightarrow E$ ) synapses such that the firing response of $E$ neurons in layer 4 is strong to particular orientations and not others. The results show that the $E$ neurons in layer 4 develop preferential firing to certain orientations and not to others (Figure 6). This illustrates that there was an emergence of OSMs with characteristic iso-orientation domains and fractures in the orientation gradient maps. However, there were no point singularities indicating the absence of any pinwheel-like formations. There were also no linear zones formed.

The feedforward inputs triggered by spontaneous background spiking activity in the LGN results in the excitation of layer 4 neurons via the geniculocortical synapses (i.e., LGN $\rightarrow E$ synapses). The synaptic inputs originating from LGN can be partitioned into two groups based on the origin of LGN neurons: left and right 

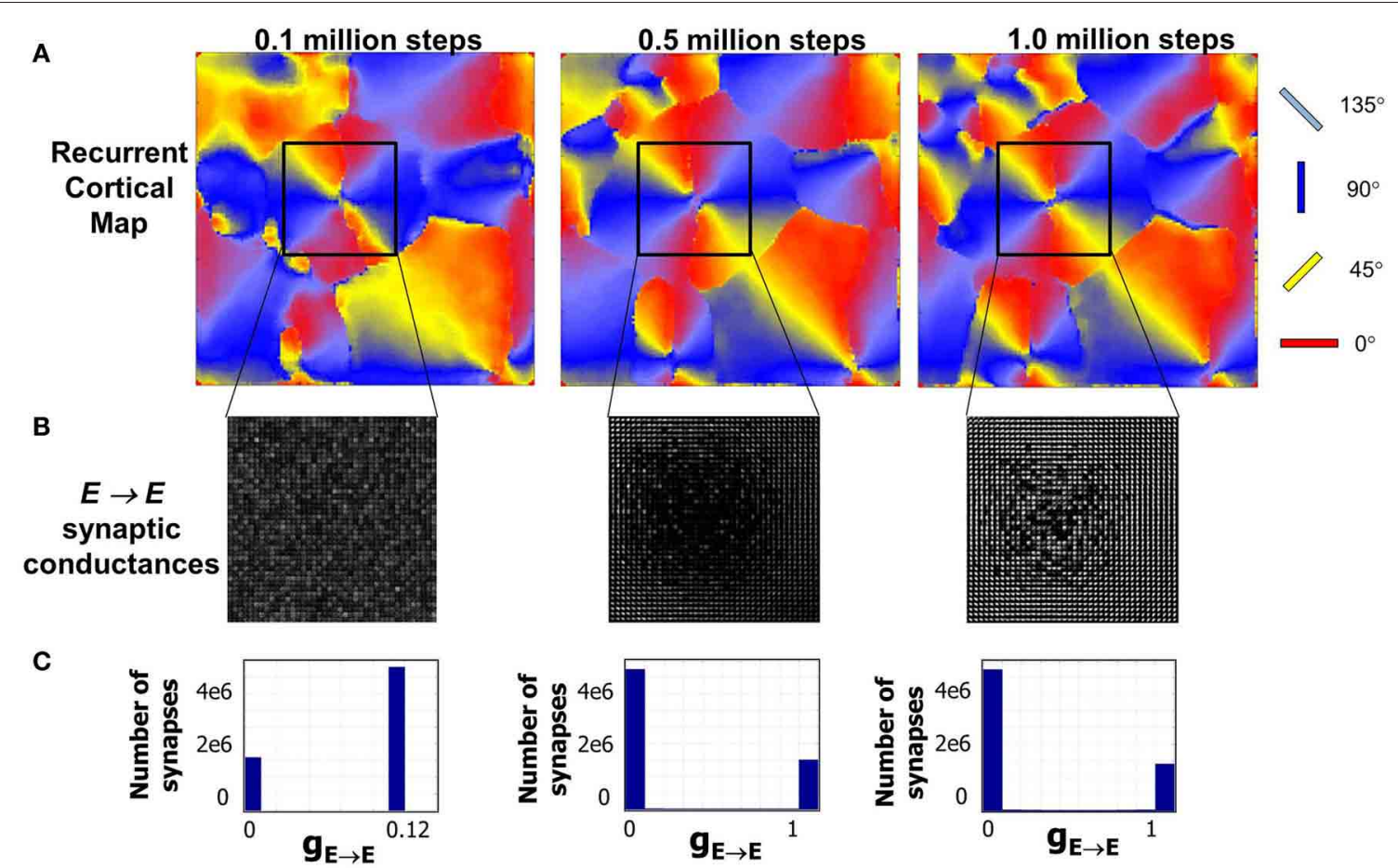

FIGURE 5 | Evolution of RCMs during Phase 1 at three different stages of development. (A) After 0.1 million steps of simulation the RCM is well formed but not completely stable. The second stage after 0.5 million steps of simulation shows the emergence of structure in local synaptic connectivity between the $E$ neurons in layer 4 (see "Materials and Methods"). The RCMs are stable after 1 million steps. (B) The synaptic conductances within the pin wheel for a $40 \times 40$ neighborhood (black square) is shown where there is a clear lack of appearance of structure after the first stage. After a 1 million steps of simulation, the RCM is well formed and the synaptic conductances show well developed structure except at the pin wheels. (C) The histogram of the synaptic conductances in the $E \rightarrow E$ synapses of layer 4 shows that the initial histogram of synaptic conductance values is bimodal with most of the synapses fixed at a value of 0.12 . As the RCM evolves, this bimodal distribution changes due to competition between $E \rightarrow E$ synapses that is caused by STDP. This competition creates a sparse network with a majority of the synapses becoming zero while a fewer set of $E \rightarrow E$ synapses are fully potentiated to 1.0 . eyes (Figure 7A). Initially the synapses are all of equal strength (i.e., no bias) such that the $E$ neurons in layer 4 respond equally to spikes from LGN neurons corresponding to both the eyes (Figure 7C). However, noisy background spiking activity in LGN causes STDP to select some synapses to potentiate while others to depress depending on temporal correlations among the spikes that impinge on the $E$ neurons in layer 4 . This process results in introducing a bias in the synaptic strengths from LGN $\rightarrow E$ neurons in layer 4 due to STDP induced competition among all geniculocortical synapses at any given $E$ neuron. As a result, the strength of geniculocortical synapses from one eye ends up being more than from the other eye (Figure 7A) and thus $E$ neurons in layer 4 begins to develop eye selectivity. The eye selectivity of $E$ neurons across layer 4 manifests as an ODM (Figure 7D). This early experience-independent formation of ODM while balanced (i. e., number of $E$ neurons that are selective to the left and right are equal) is still fragmented without any contiguous patches of neurons as found in the visual cortex of several species. This is because in our model, the LGN neurons are primarily stimulated via background activity that has no temporal or spatial contiguity during Phase 1 . The mean value of the difference in synaptic conductance between the geniculocortical synapses from both eyes increases slowly (Figure 7B). However, the standard deviation of the difference is higher than the mean implying that many of the $E$ neurons have a very small difference in synaptic conductances while a few have a much larger difference. This measure shows that the ODMs are not really stable during the Phase 1.

\section{PHASE 2: LATE EXPERIENCE-INDEPENDENT ODM AND OSM REFINEMENT}

In the second phase of development, in addition to the basic thalamocortical circuit of Phase 1, the LGN is now connected to two groups of RGC corresponding to the two eyes (Figure 1). The RGC inputs to LGN represent internally generated spikes in the form of retinal waves ("Materials and Methods") that provide a robust signal to drive activity in both the LGN and in layer 4 of V1. Such spontaneous activity has been implicated in the development of ODMs and retinotopy in V1 (Mooney et al., 1996; Butts, 2002; Godfrey and Swindale, 2007; White and Fitzpatrick, 2007; Huberman et al., 2008; Feller, 2009). An example of a retinal wave generated for a given eye with $N=10$ is shown in Figure 8A. The retinal waves after 300 and 200,000 waves are shown in Figures 8B,C, respectively. It can be noted that by the end of 200,000 waves, all the RGCs have been selected as initiation sites and the distribution of RGC activity resembles a Gaussian random field. 


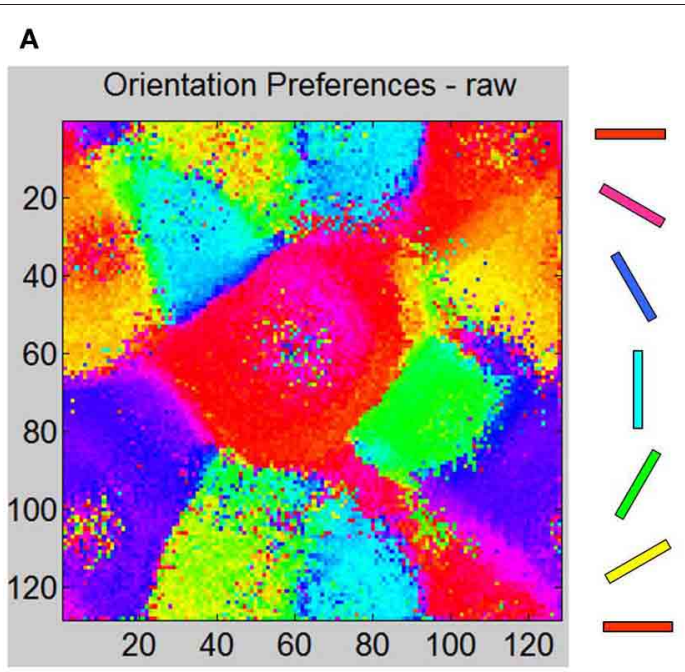

C

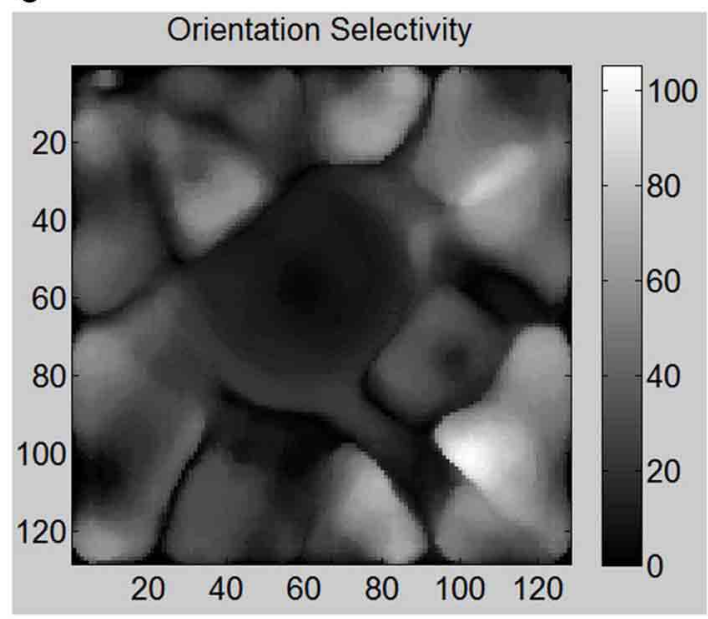

FIGURE 6 | Various aspects of formed OSM at the end of Phase 1 including orientation gradients, orientation selectivity, and orientation preferences of neurons are shown here. (A) The oriented bar stimuli are provided as input to the LGN neurons and the raw firing rates of the $E$ neurons in layer 4 are measured ("Materials and Methods"). (B) The smoothed responses (see "Materials and Methods") of orientation preferences is computed and plotted in color. The oriented bars on the right provide the cyclic color code ranging from $0^{\circ}$ to $180^{\circ}$. (C) Orientation selectivity of each $E$ neuron is indicated using a grayscale map. The brighter colors indicate high selectivity where those $E$ neurons in layer 4 respond sharply to a very narrow range of orientations and vice versa. The color scale shows the magnitudes of responses in a relative fashion. For example, neurons in the neighborhood of neuron at $(110,100)$ show strong
B

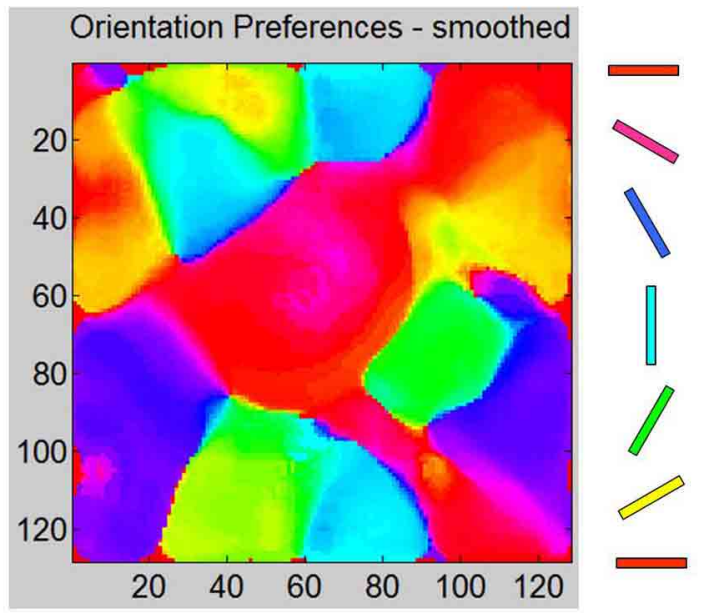

D

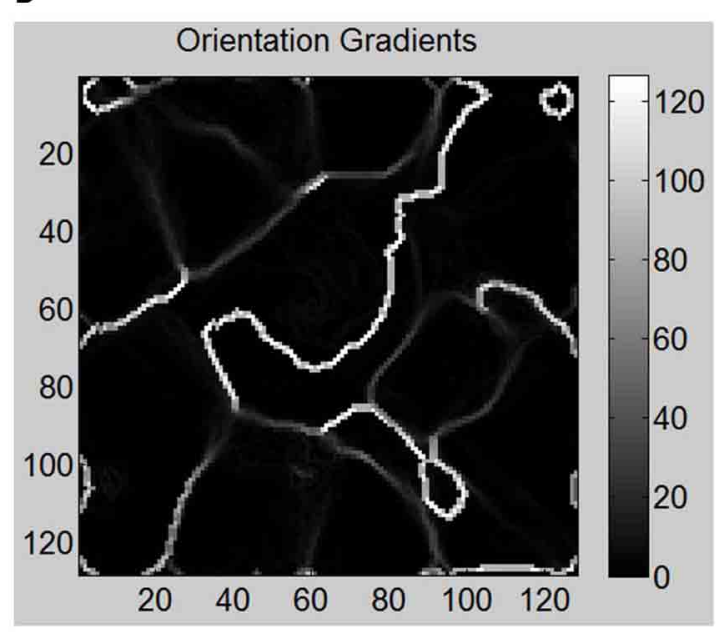

selectivity (score of 110 ) to only $120^{\circ}$ but not to other orientations while a neuron at $(80,50)$ shows a weak selectivity (score of 8 ) to all orientations including its preferred orientation of $150^{\circ}$. (D) The absolute magnitude of the orientation gradients at each $E$ neuron is shown here. Here lighter values indicate high gradients (closer to $90^{\circ}$ ) while black indicates there neighborhoods have similar orientation preferences and thus no gradient at all. Orientation selectivity and orientation gradients are linked such that regions of high selectivity typically have low gradients while regions of low selectivity have high gradients in a manner qualitatively consistent with the data from the Blasdel paper. The discontinuous changes either occur alone (singularities), or they group together along lines (fractures). While there are many lines of fracture in this phase, there are no singularities, linear zones or pinwheels.
In this model, the spikes generated by retinal waves are transmitted via geniculocortical synapses to activate the $E$ neurons in layer 4 . It should be noted that our model assumes that in this phase of development, there is no separation of RGCs into ON and OFF ganglion cells (Myhr et al., 2001; Huberman et al., 2008). The distribution of the geniculocortical synapses to $E$ neurons is sharpened further due to STDP and the resulting ODM shows more distinct patches selective to a given eye compared to ODMs in Phase 1 (Figure 9A).
The emergence of ODMs was also analyzed based on lesion studies. There are animal studies that suggest, for example, that retinal wave disruption caused by intraocular injection of epibatidine reduces firing of the RGC thereby affecting the development of eye-specific retinogeniculate projections and eventually the development of functional maps as well (Wong, 1999; Feller, 2009). In our model, we qualitatively simulate the disruption of retinal waves by cutting off neural activity in a percentage of neurons in the right eye of the LGN. The resulting RCMs, 
A

If $\sum_{i} w^{\text {left }}{ }_{i j}>\sum_{i} w^{r i g h t}{ }_{i j} \Rightarrow \mathrm{j}=$ Left

If $\sum_{i} w^{\text {right }}>\sum_{i} w^{\text {left }}{ }_{i j} \Rightarrow \mathrm{j}=$ Right

If $\sum_{i} w^{\text {right }}{ }_{i j}=\sum_{i} w^{\text {left }}{ }_{i j} \Rightarrow \mathrm{j}=$ Binocular

LGN

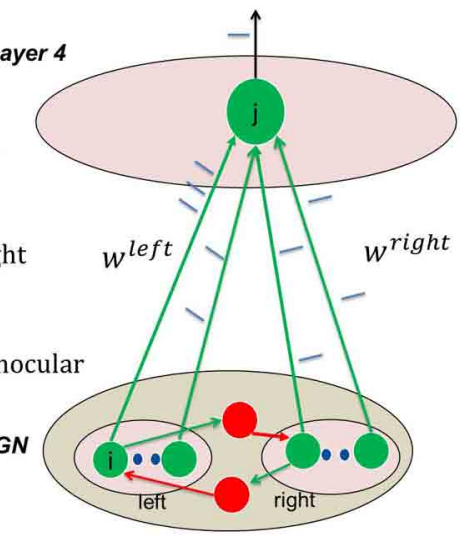

B

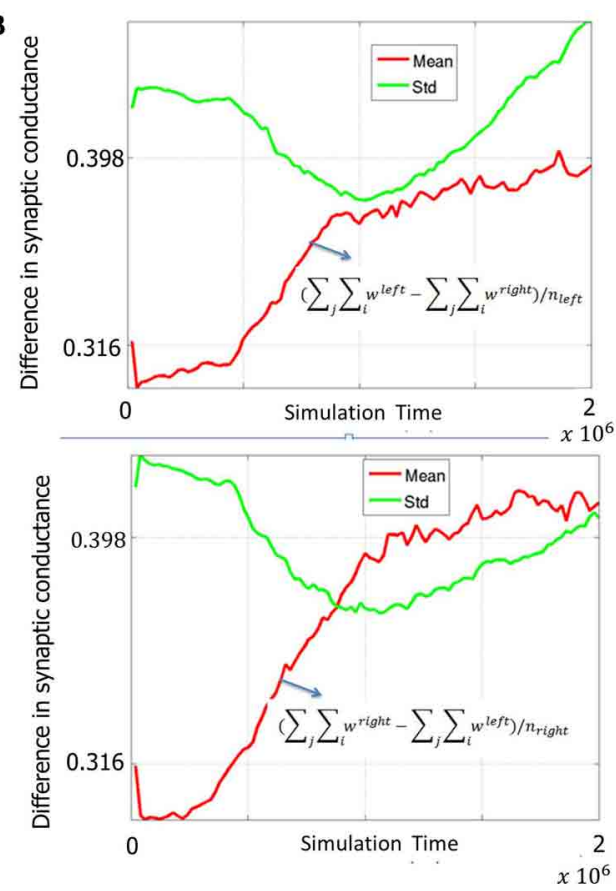

C

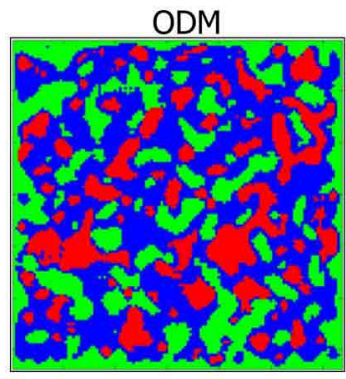

1000 steps

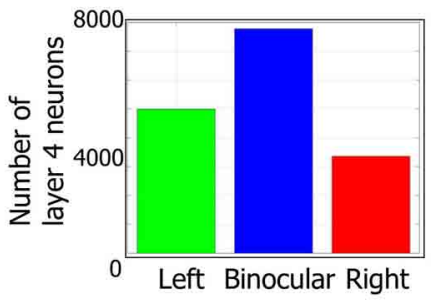

D

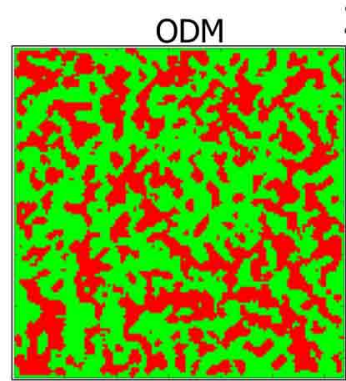

\section{2 million steps}

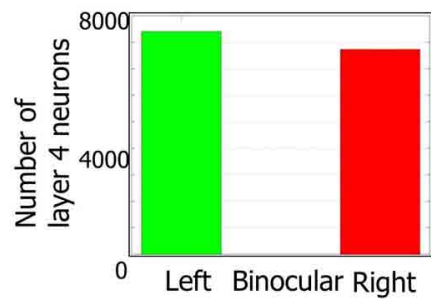

FIGURE 7 | Formation of ODMs during Phase 1. (A) The methodology of constructing the ODMs is outlined here (see "Materials and Methods"). The synaptic conductance changes induced due to STDP at the geniculocortical synapses from the LGN neurons corresponding to each eye are tracked over time. If the $E$ neuron in layer 4 has a stronger set of afferents from the LGN from the left eye compared to the right eye, then the $E$ neuron is labeled as "left" and color coded as green in the ODM. The exact opposite scenario results in the $E$ neuron labeled as "right" and color coded as red in the ODM. If there is a tie (as in the beginning), then the $E$ neuron is labeled as "binocular" and color coded as blue in the ODM. (B) The sum of the synaptic conductances from the LGN neurons corresponding to the left eye is compared against the sum of the synaptic conductances from the LGN neurons corresponding to the right eye at each $E$ neuron. Each of these neurons are labeled as "left" or "eye" as described above. Then the mean and standard deviation of the difference between left and right eye afferent synapses for the neurons (nleft) labeled "left" is computed and plotted in a semilog format with the ordinate plotted in log scale while the abscissa data is plotted in regular scale. It can be seen that the mean value of the difference increases slowly.
However, the standard deviation of the difference is higher than the mean implying that many of the $E$ neurons have a very small difference in synaptic conductances while a few have a much larger difference. Similar behavior was observed for the right eye as well. This measure shows that the ODMs are not really stable during the Phase 1 in our model. (C) Early ODM appears to have several binocular $E$ neurons since all the geniculocortical synapses are initialized with the same synaptic strength and there have not been sufficient inputs to alter the synaptic strengths via STDP. (D) At the end of Phase 1, the $E$ neurons in the ODM shows eye selectivity with an even split of neurons becoming selective to one of the two eyes. There are no more binocular neurons. The ODM, however, appears fragmented with no large contiguous areas of neurons showing preference to one eye and not the other. Instead it has lots of small contiguous areas of various sizes. This is due to random stimulation of the LGN neurons with background activity with no temporal or spatial contiguity during Phase 1. The STD and mean data in (B) also provides further support to this basic phenomenon during this phase. However, this improves during the second and third phases of development as shown in Figures 10, 12 when there is more structure in the input data.
OSMs, and ODMs (Figures 9B-D) are compared against the case of normal development (Figure 9A). In our model, this bias emerges due to the competitive nature of the STDP rule. When more inputs are received from the left eye, there is a higher probability for the geniculocortical synapses from the left eye to cause a post-synaptic spike. This in turn implies that the geniculocortical synapses from the left eye are going to potentiate a lot more than those from the right eye and thus the $E$ 

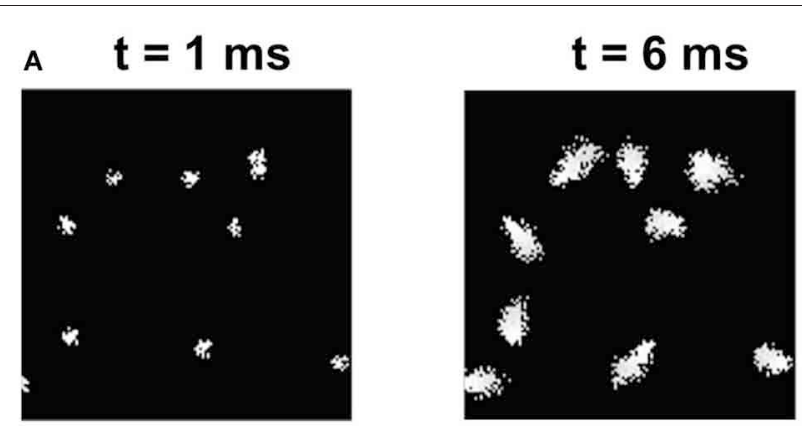

B $\quad 300$ waves

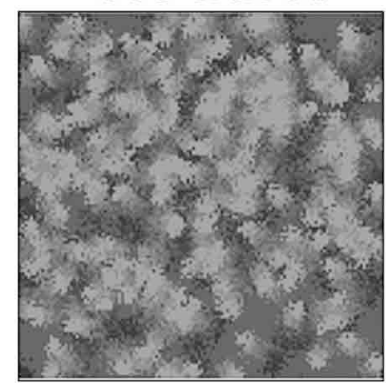

c 200,000 waves

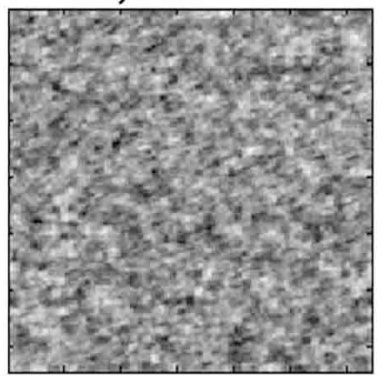

FIGURE 8 | The retinal wave model. (A) Shows $128 \times 128$ RGCs from one retina. After $1 \mathrm{~ms}$ of spontaneous firing of RGCs, there are 10 sites that are randomly initiated to generate spikes. These sites create further activity among neighboring RGCs to propagate a wave of spikes as shown for $t=6 \mathrm{~ms}$ and $t=10 \mathrm{~ms}$. Each such spontaneously initiated wave activity is terminated at the end of $10 \mathrm{~ms}$. The $128 \times 128$ retinal wave image is donw-sampled to a $48 \times 48$ image and provided as input to the LGN. (B) The superposition of all spike activity after 300 retinal waves shows a good spatial distribution of spike activity covering all parts of the retina. (C) The late experience-independent phase in our model lasts for around 200,000 retinal waves when all the RGCs in the retina are activated at least once and the spiking activity of RGCs resembles a Gaussian random field-like distribution. neurons in layer 4 become more selective to spikes from the left eye.

RCM development is only affected in a minor fashion due to lesions to the LGN cells because RCM formation is primarily dictated by the $E \rightarrow E$ synaptic conductance maps while ODM and OSM formation is dictated by both LGN $\rightarrow E$ and $E \rightarrow E$ synapses. Since the changes in inputs from retinal waves drive the geniculocortical synapses, the spikes in the $E$ neurons in layer 4 are primarily caused by the LGN. So, the synaptic changes in geniculocortical synapses (which affects ODM and OSM) are more dramatic than in the cortico-cortical synapses (which affects OSM and RCM).

The ODMs formed in Phase 2 show more contiguous patches of eye selectivity (Figure 10A) compared with Phase 1. The mean and standard deviation of difference in synaptic conductances (Figure 10B) show a more clear separation on the semi-log plot indicating that the ODM formed in Phase 2 is more stable than in Phase 1. The orientation tuning of $E$ neurons in layer 4 during Phase 2 was evaluated by stimulating the LGN neurons for both eyes using the same oriented bar stimuli (Figure 6) during Phase 1. The results show that the $E$ neurons in layer 4 develop more well-defined iso-orientation domains (Figure 10E) and fractures (Figure 10C) in Phase 2 compared to Phase 1. This change is caused by STDP due to a spike inputs from the geniculocortical synapses in Phase 2 due to the retinal waves that have more spatial and temporal contiguity compared to noisy background level activity in Phase 1. The peak firing response of $E$ neurons in layer 4 are on the average larger than in Phase 1 as indicated by the larger dynamic range in orientation selectivity maps (Figure 10D). This shows that the E neurons in Phase 2 have developed sharper orientation selectivity and the OSM is actively evolving due to activity-dependent plasticity. There are also three clear pinwheel-like patterns that emerge in this stage as depicted in black circles (Figure 10E) where the orientation preferences rotate continuously through $\pm 180^{\circ}$ along circular paths. However, there were no corresponding point singularities that could be extracted from the orientation gradient due to the limitations of the methodology used (see "Materials and Methods").

\section{PHASE 3: EXPERIENCE-DEPENDENT OSM AND ODM REFINEMENT AND MAINTENANCE}

For the third phase of the developmental process, the model is exposed to sensory experience in the form of images from a database of real-world images from the Caltech 101 database (see "Materials and Methods") to study the effects of experiencedependent plasticity (Yao and Dan, 2001) on the refinement of OSMs and ODMs. In our model, the RGCs in this phase are assumed to be segregated into ON and OFF neurons as evident in animals after eye opening (Koehler et al., 2011). The ON and OFF regions each contain $48 \times 24$ neurons. The $\mathrm{ON}$ neuron responses are encoded as spikes (see "Materials and Methods"). The OFF 


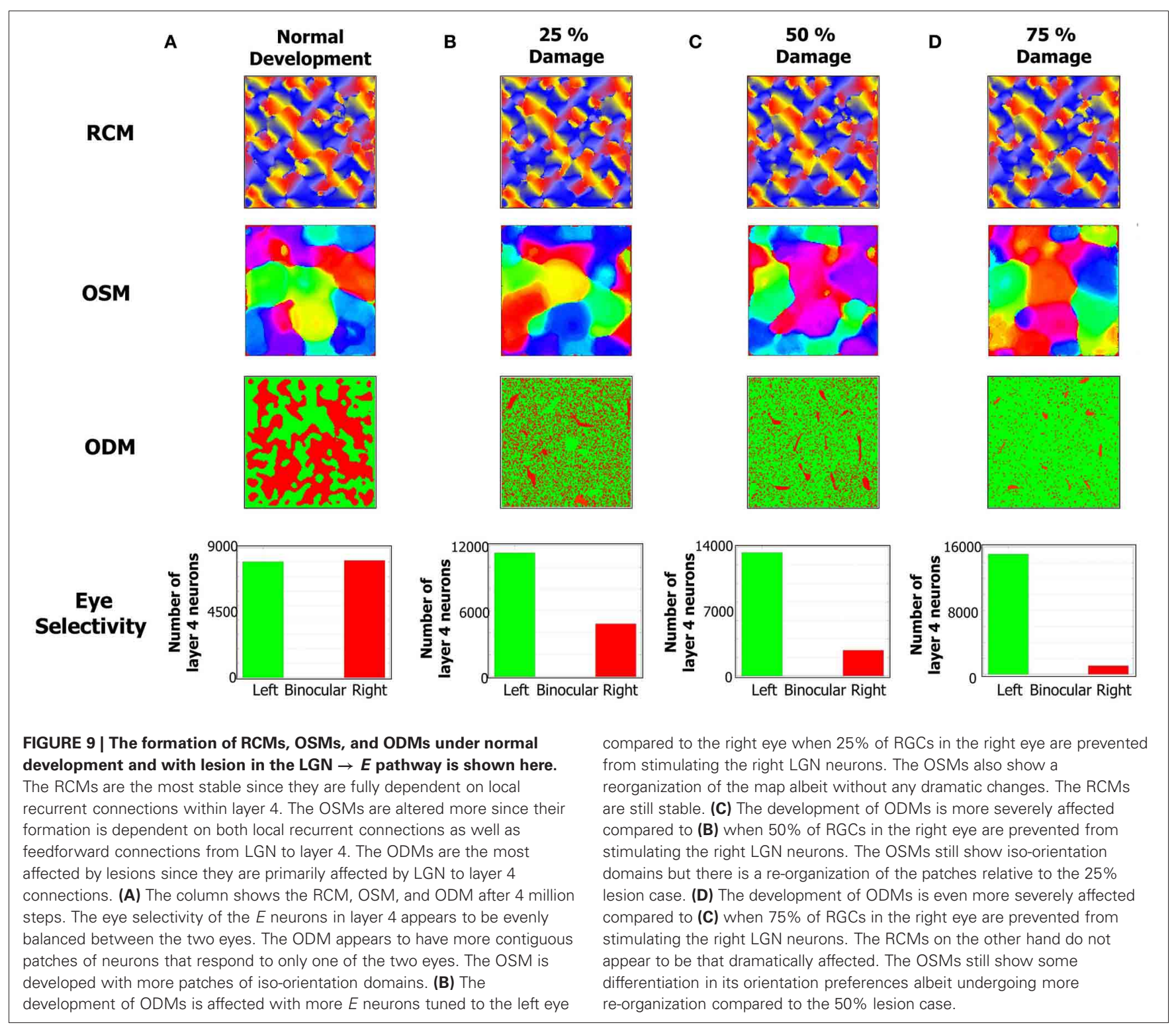

neuron responses are computed by taking the inverse of the ON image and then rectifying the image so that there are no negative values (Figure 11). The resulting image is encoded as spikes (Equation 11).

The ODMs formed in Phase 3 show contiguous patches of eye selectivity (Figure 12A) in a manner similar compared with Phase 2. The mean and standard deviation of difference in synaptic conductances (Figure 12B) show an even more clear separation on the semi-log plot indicating that the ODM formed in Phase 3 is more stable than in Phase 2. The results also show that $E$ neurons in layer 4 are more finely tuned to particular orientations compared to Phase 2 (Figure 12E). The dynamic range of the orientation selectivity map (Figure 12D) is larger than in Phase 2. There are four distinct pinwheel-like patterns that emerge after learning in Phase 3 (Figure 12B) but do not have any point discontinuities in the orientation gradient maps at the centers of these pin wheel patterns. The ODM maps are also more refined and develop noticeable contiguous regions of eye selectivity. The interesting aspect here is that the RCMs seem to have stabilized in Phase 2 and appear qualitatively similar between Phase 2 and Phase 3 while the ODM and OSM continue to undergo refinements after exposure to the images from the Caltech 101 database for over 6 million steps. This is qualitatively consistent with some experimental observations (Chapman et al., 1996; Crair et al., 1998) where the orientation tuning responses change with environmental stimuli.

\section{STABILITY OF FUNCTIONAL MAPS}

The network was analyzed for stability across all three phases of development. In Phase 1, the balance in synaptic currents driving neurons of layer 4 for this phase of development was measured by computing the difference between excitatory and inhibitory currents at each $E$ neuron by averaging across a time window 
A

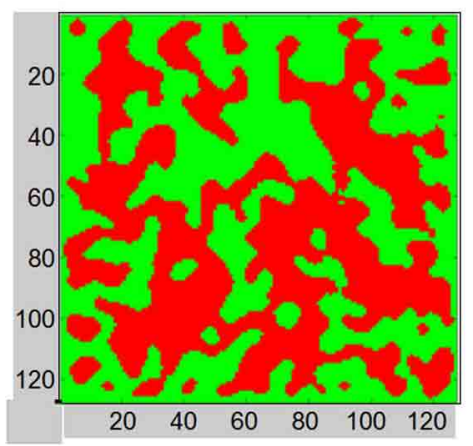

C

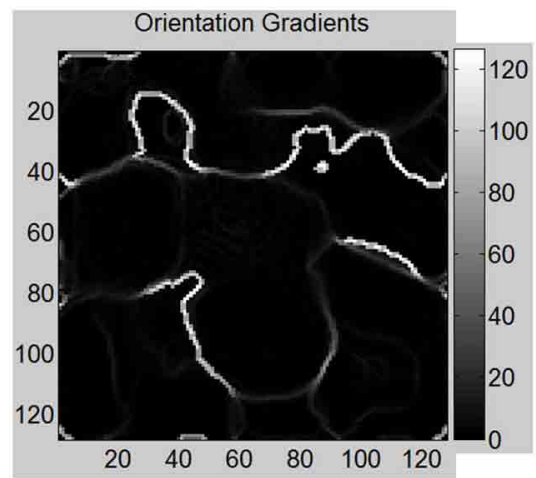

B

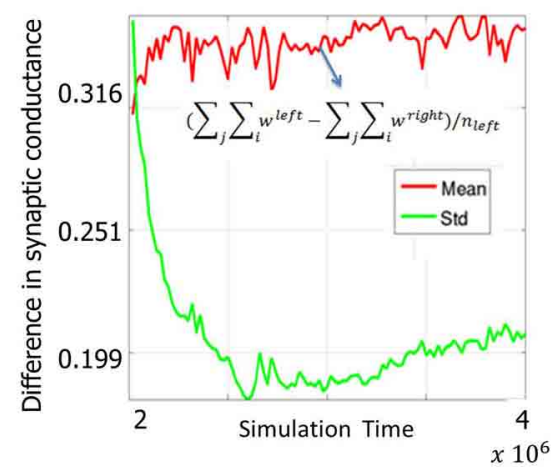

D

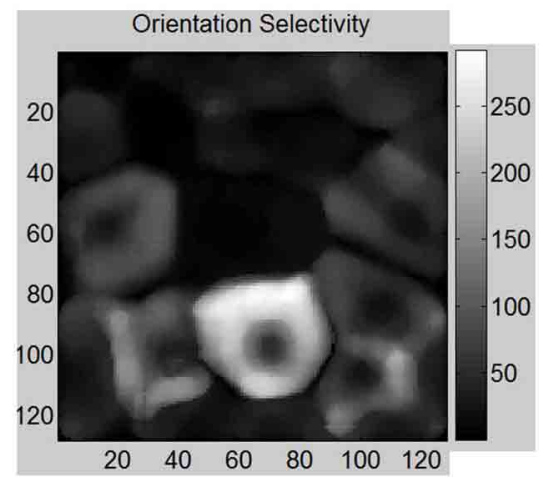

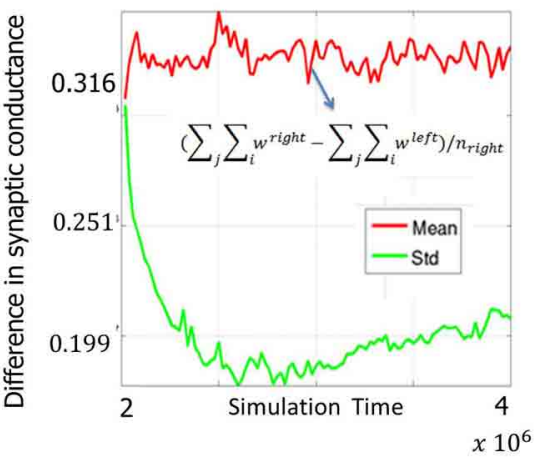

E

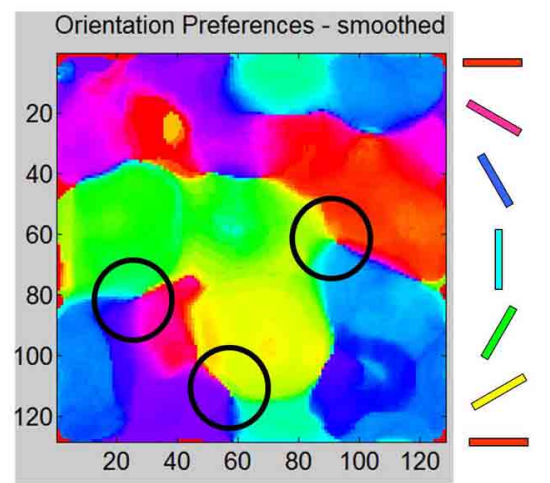

FIGURE 10 | ODM and OSM formation during Phase 2. (A) The ODM appears to have large more contiguous patches of eye selectivity compared to the output after Phase 1. (B) This maturity in ODM formation is caused by more stability in the divergence between the mean and standard deviation calculations in the semilog plots shown here. This divergence is very clear unlike in Figure 7. The mean and standard deviation also seem to stabilize in the later parts of Phase 2. (C) The absolute magnitude of the orientation gradients at each $E$ neuron shows singularities and fractures. Here white indicates high gradient values while black indicates no gradient at all. The gradients image show several fractures in the data. The average fraction of the total synaptic drive at each $E$ neuron selective to a given eye was also calculated. For example, for the "left" $E$ neurons, $\left(\sum_{i} w^{\text {left }}-\sum_{i} w^{\text {right }}\right) /\left(\sum_{i} w^{\text {left }}+\sum_{i} w^{\text {right }}\right)$, was $\sim 71 \%$. Similarly, the fraction was $\sim 70 \%$ for the "right" $E$ neurons at the end of Phase 2. (D) Orientation selectivity shows brighter colors that indicate high selectivity with those $E$ neurons in layer 4 respond to a very narrow range of orientations and vice versa. The color scale shows the magnitudes of responses in a relative fashion. For example, neurons in the neighborhood of neuron at $(85,80)$ show strong selectivity (score of 270) to only $30^{\circ}$ but not to other orientations while a neuron at $(60,60)$ shows a weak selectivity (score of 3 ) to all orientations including its preferred orientation of $60^{\circ}$. (E) The smoothed orientation preference map shows iso-orientation domains and three weakly formed pinwheels marked by the three black circles. We call these weakly formed pin wheels since they are not corroborated by singularities in the orientation gradient maps. This is because the smoothing operation on the Cartesian images removes the spurious edges created by noisy neuron responses while also removing any trace of the singularities as well. However, close inspection shows that there are three locations marked with black circles where the orientation preferences rotate continuously through $\pm 180^{\circ}$ along circular paths. We refer to these patterns as a pinwheel-like pattern. It should be noted that there are no clear appearance of point discontinuities in the orientation gradient maps to corroborate the pin-wheel centers within these pinwheel-like-patterns that clearly appear in animal data. of $200 \mathrm{~ms}$. Simulations show that the net current during this phase moves from an initial bias toward excitation to a more negative bias toward inhibition that slowly reaches a steady state value (see red line in Figure 13A). The negative bias shows that on average the influence of inhibition is stronger than excitation so as to compensate for the imbalance in the number of $E$ to $I$ neurons in layer 4 . This dynamic ensures that the firing rates of the neurons are low and conducive for learning the RCMs and functional maps.

The distribution of synaptic conductances was also tracked dynamically throughout the learning process by computing the difference between the normalized distribution of synaptic conductances $(E \rightarrow E, E \rightarrow I, I \rightarrow E$, and $I \rightarrow I$ as shown in Figure 14) using the KL measure (Equation 17) once every 5000 simulation steps (or $5 \mathrm{~s}$ ). This dynamic captures a measure of stability in the learning process during development. The results show that all the four types of conductances slowly reach a steady state at the end of Phase 1 with the KL divergence showing very small fluctuations.

The network exhibits a better balance between excitation and inhibition progressively as a function of development (Shapley et al., 2003; Okun and Lampl, 2008) (see Figure 13). The stability 


\section{Caltech 101 Image}

A

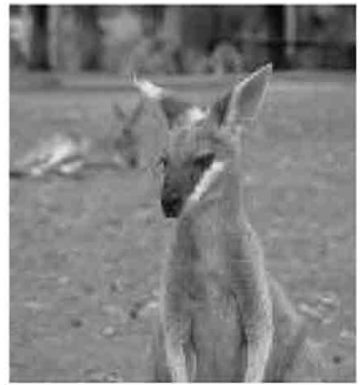

Down sampled Image
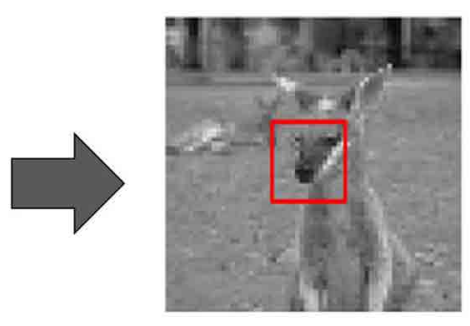

ON
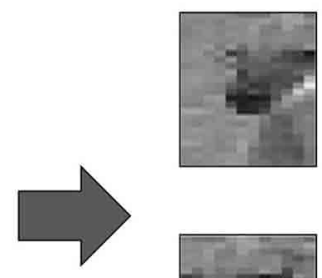

\section{OFF}

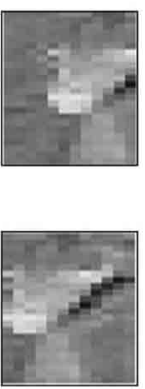

Left

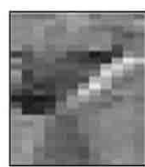

Right
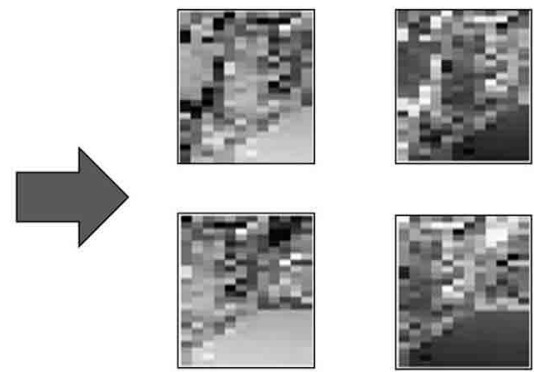
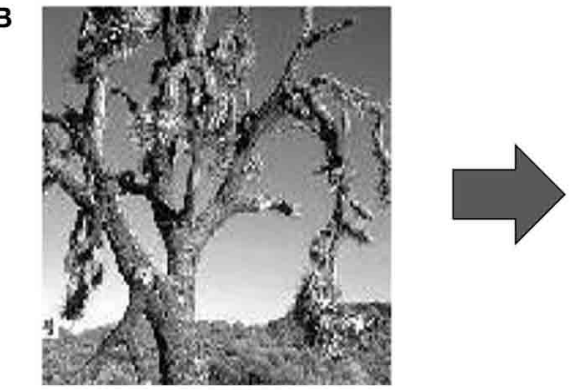

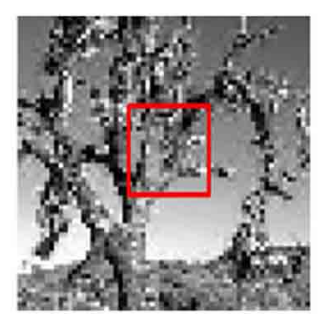

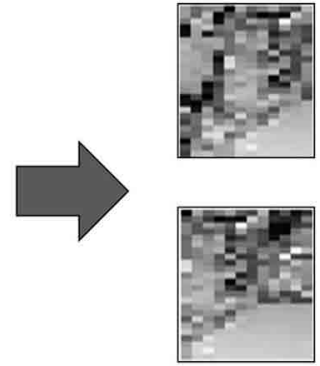

FIGURE 11 | Natural stimuli during Phase 3. (A) The input image from the Caltech database is down sampled from a $320 \times 200$ image to a $128 \times 128$ image and pixels from within a $48 \times 48$ window from the center of the sub-sampled image (red box) are used to create the stereo pair. The resulting image for the right eye is obtained by shifting the fovea to the right and applying a scaling factor of 1.025 on the left image. The resulting stereo pair is finally processed to generate ON and OFF images for each eye (see "Materials and Methods"). (B) A second example of an image from the Caltech 101 database using the same scaling and pixel shift as in (A) is used for extracting the down sampled ON and OFF images. The appearance of the pixels in the ON and OFF images for both examples has similar statistics in terms of the contrast and oriented edges. of the formed RCMs in the second phase of development is measured using KL divergence in synaptic conductances (Figure 14). The results show that the network is progressively more stable during development since the KL divergence is lower progressively for all four types of synaptic conductances in layer 4 .

The OSMs and ODMs undergo refinement throughout the three phases of development. The primary cause for the refinement is the change in the nature of LGN inputs from random activity in Phase 1 to retinal waves in Phase 2 to natural stimuli in Phase 3. At the end of Phase 3 (i. e., after over 5.5 million steps of natural image stimuli) the OSMs and ODMs appear to stabilize such that the orientation responses of neurons in layer 4 no longer shift as observed earlier (compare Figures 6, 10, and 12).

It is known that OSMs and ODMs continue to undergo noticeable refinements if there is substantial change in the input environment (Blakemore and Cooper, 1970; Sengpiel et al., 1999; Krelle et al., 2011). We conducted an experiment to verify if this occurs in our model. We created a set of new flag patterns primarily consisting of horizontal and vertical bars (Figure 15A). This stimulus is considerably different from the Caltech 101 database images since they do not provide contrast information in any other direction except $0^{\circ}$ or $90^{\circ}$. These inputs stimuli were provided as stereo inputs to the LGN ("Materials and Methods"). Despite repeated presentations of such stimuli ranging from short $\left(T_{F}<1 \mathrm{~s}\right)$ to longer durations $\left(T_{F}=10 \mathrm{~s}\right)$, the OSMs and ODMs are not affected and remain stable (Figure 15B). This is due to an exquisite balance in currents created by the E-STDP and I-STDP plasticity mechanisms where any instantaneous imbalance in currents is rapidly compensated by plasticity to restore the balance (Figure 13).

If there is a volley of high-frequency spikes due to stimuli during such short durations that cause the $E$ neurons to spike more rapidly, the inhibitory plasticity for both feedforward and recurrent synaptic connections enables the system to increase the synaptic conductance $z$ of the inhibitory synapses. This results in increased inhibitory currents to the active $E$ neurons thereby removing the imbalance (Vogels et al., 2011). Thus E-STDP and I-STDP enables the stable maintenance of OSMs and ODMs despite continuous plasticity.

We also applied the same flag patterns with horizontal and vertical bars for a very long duration $\left(T_{F}=5000 \mathrm{~s}\right)$. The OSMs and ODMs do see changes (Figure 15C) after this long exposure reflecting that only prolonged and consistent presentations 
A

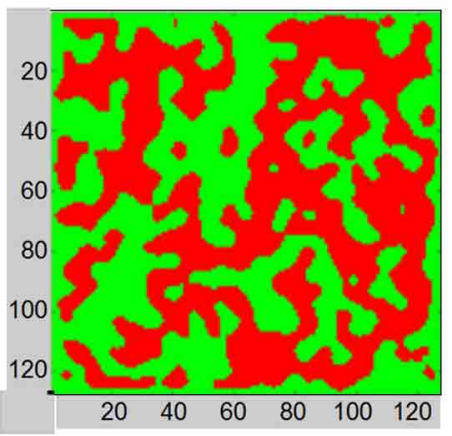

C

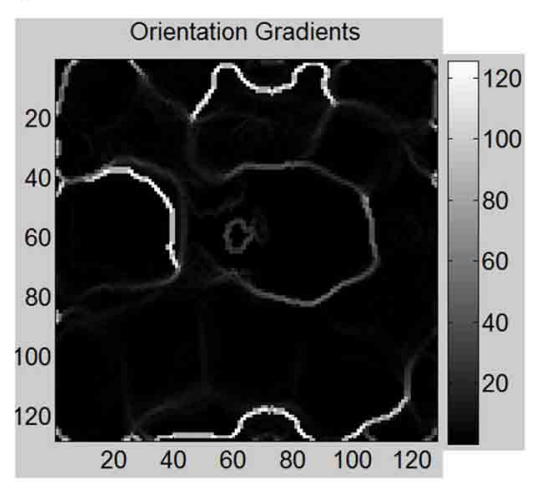

B

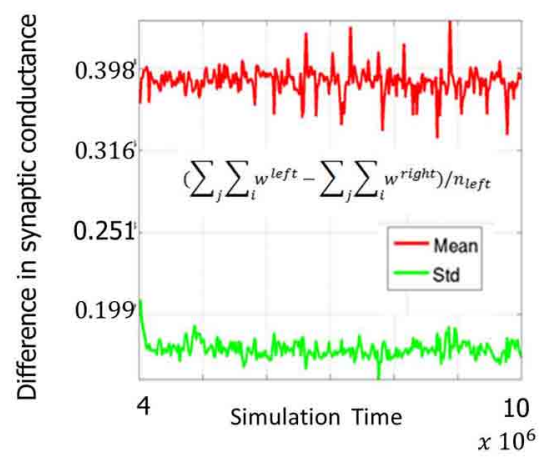

D

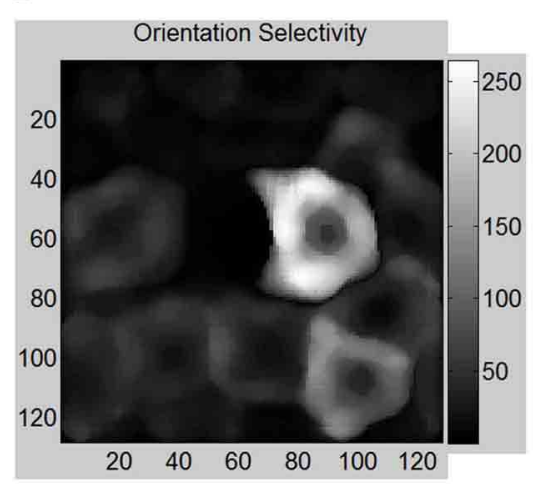

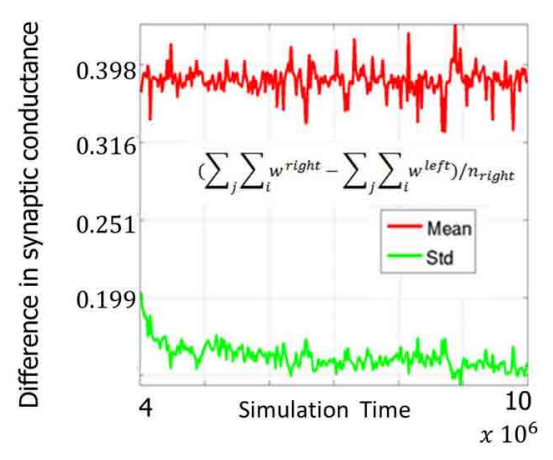

E

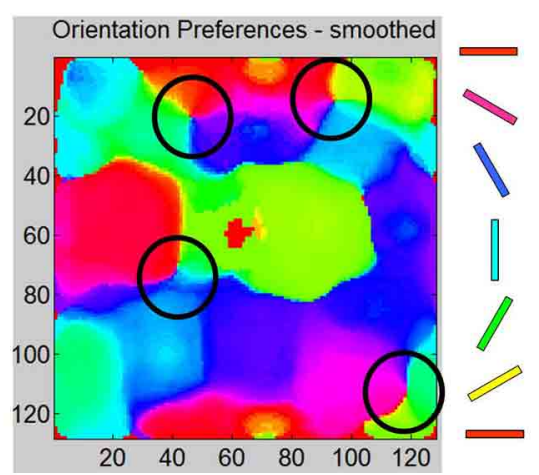

FIGURE 12 | Functional maps summary and orientation selectivity of neurons in OSMs during Phase 3. (A) Comparison of functional map development during all three phases shows the progressive refinement of the ODM, RCM, and OSM. The ODM is stable and shows well-defined and contiguous patches of eye selectivity. (B) The mean and standard deviation parameters as described in Figures 7, 10 show more divergence and stability compared to Phase 2 The average fraction of the total synaptic drive for the "left" E neurons was 123\% and 124\% for the "right" $E$ neurons at the end of Phase 3. (C) The resulting orientation gradient maps show well-defined fractures but no singularities. (D) The orientation selectivity has similar characteristic to that of Phase 2 except that the peak magnitude of selectivity showing more sharpness (i.e., higher magnitude). (E) The orientation preference maps show clearly defined iso-orientations and four weakly formed pinwheels marked by four black circles. of stimuli can change the orientation tuning response of the $E$ neurons in layer 4 . This change is again possible due to activity-dependent STDP that slowly changes the tuning properties of $E$ neurons during the small windows of opportunity where $E$ neurons in layer 4 spike despite operating in a well-balanced regime between excitation and inhibition. Since these spikes are sparse, our model requires prolonged periods of stimuli exposure to effect changes in the stabilized ODM and OSM. These results qualitatively agree with experimental results (Blakemore and Cooper, 1970; Sengpiel et al., 1999; Krelle et al., 2011) that suggest that influence due to environmental stimuli can affect functional maps in V1. It also shows that the number of $E$ neurons that respond to $90^{\circ}$ (blue regions) or $0^{\circ}$ (red regions) increases by $25 \%$ or more compared to OSM at the end of 10 million steps (Figure 15C). This is also qualitatively consistent with observations in the visual cortex (Sengpiel et al., 1999). It is interesting that RCMs are not affected much because geniculocortical synapses are affected more by new inputs from LGN compared to cortico-cortical synapses.

\section{DISCUSSION}

The proposed spiking model is the first to cover the three developmental phases during the formation of OSMs and ODMs with continuous synaptic plasticity in the form of STDP. The model offers a biologically plausible explanation for this formation in which E-STDP and I-STDP at the excitatory and inhibitory synapses, respectively combine to enable the development and maintenance of these functional maps. This is consistent with recent models that suggest that cortical reorganization is reliant on spike timing (Song et al., 2000; Young et al., 2007). It is also consistent with a recent model that suggests that inhibitory plasticity could play a key role in the formation and maintenance of functional cortical circuitry (Vogels et al., 2011).

It is possible for our model to demonstrate OSM and ODM formation directly via external simulations (i.e., experience) and skipping the two experience-independent phases. However, our simulations demonstrate that STDP based learning driven by spontaneous intra-cortical spiking activity can result in the formation of orientation maps and ocular dominance maps as 

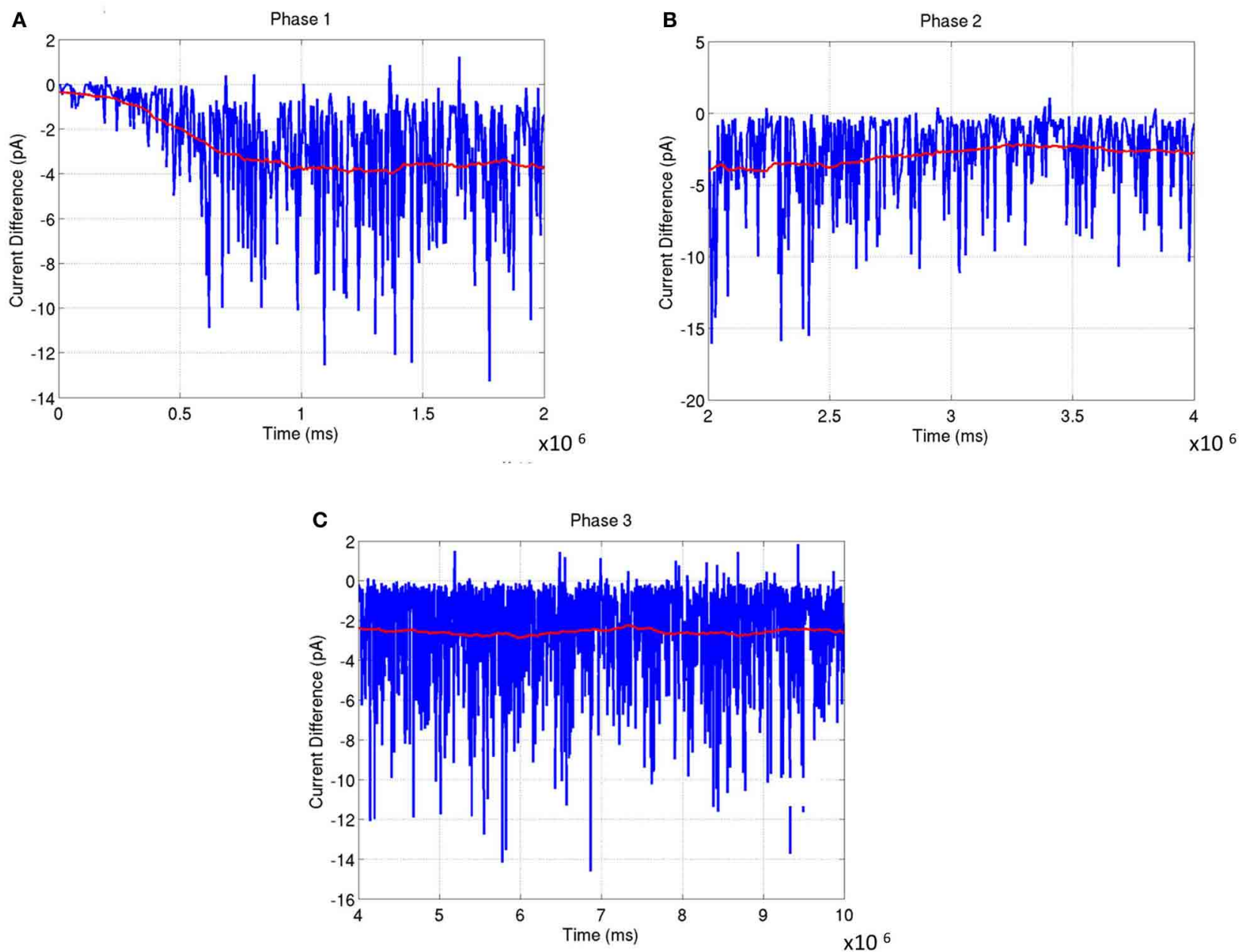

FIGURE 13 | The average synaptic current difference between excitation and inhibition is plotted as a function of developmental time. (A) Experience-independent OSM development (Phase 1).

(B) Experience-independent ODM development (Phase 2).

(C) Experience-dependent refinement of both OSM and ODM (Phase

3). In all the plots, the instantaneous current difference at each time step is shown in blue while the average current difference is shown in red. The plots show that the stability of the functional maps are correlated closely to the fact that the average current differences become progressively smaller as the maps develop. This is enabled by inhibitory plasticity and helps in preventing any rapid changes in synaptic conductances. suggested by prior research (Huberman et al., 2006; White and Fitzpatrick, 2007). In this early experience-independent phase ODMs and OSMs appear due to spontaneous activity between LGN neurons and the $E$ neurons in layer 4 . In the late activity-independent phase where the spontaneous activity in the LGN is generated via retinal waves (Butts, 2002; Godfrey and Swindale, 2007), the ODMs appear to become selectively tuned to one of the two eyes due to competition induced by E-STDP between geniculocortical synapses (Feller, 2009) thus dividing the cortical layer 4 into ocular dominance patches. The important point here is that the model qualitatively shows that there is no need for external input to enable the development of ODMs or OSMs as long as there is STDP driven plasticity in both $E$ and $I$ synapses. The influence of external inputs, however, does improve the sharpness of the tuning responses in V1.
However, the model in this study should be considered a simple model that is biologically incomplete in its complexity. It does not consider the development of retinogeniculate synapses (Chen and Regehr, 2000; Feller, 2009) nor does it model the differential sensitivities of the ON and OFF cells in their response to variations in contrast relative to mean luminance (Zaghloul et al., 2003). It also does not consider other types of plasticity found in biology such as short term plasticity (Tsodyks and Markram, 1997; Tsodyks et al., 1998) that affects population dynamics during different functional states (Mark and Tsodyks, 2012) and homeostatic plasticity (Turrigiano and Nelson, 2004; Watt and Desai, 2010). It also does not model the complexity in structure and function of neurons including multiple compartments (Hodgkin and Huxley, 1952; Izhikevich, 2004) and other forms of plasticity such as dendritic plasticity and its relation to STDP (Williams et al., 2007; Sjostrom 


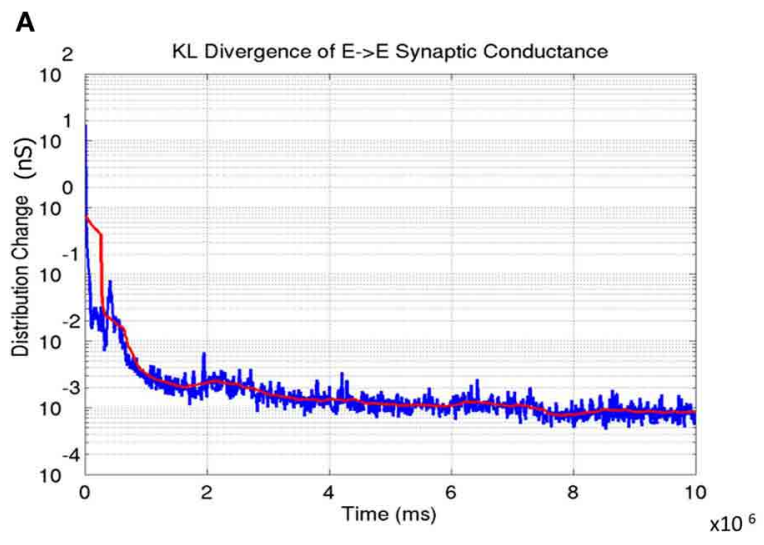

C

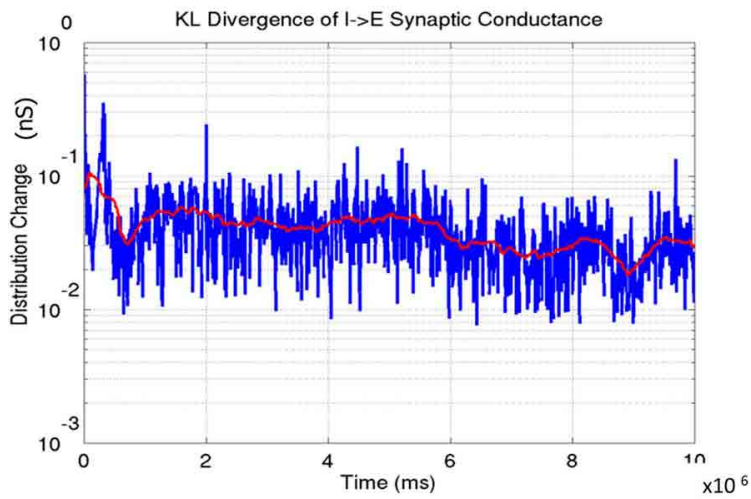

FIGURE 14 | The change in synaptic conductance distributions during the course of learning. (A) The $E \rightarrow E$ change shows a gradual decrease in the change with the lowest values in the third phase indicating stability in the formed OSM and ODMs. (B) The $E \rightarrow I$ change shows a similar trend as in (A). (C) The $I \rightarrow E$ change also shows gradual stabilization as a function of development. (D) The

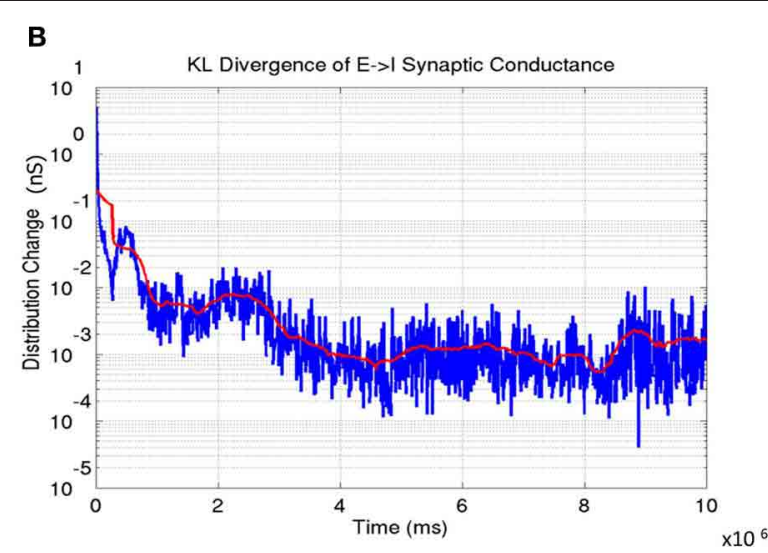

D

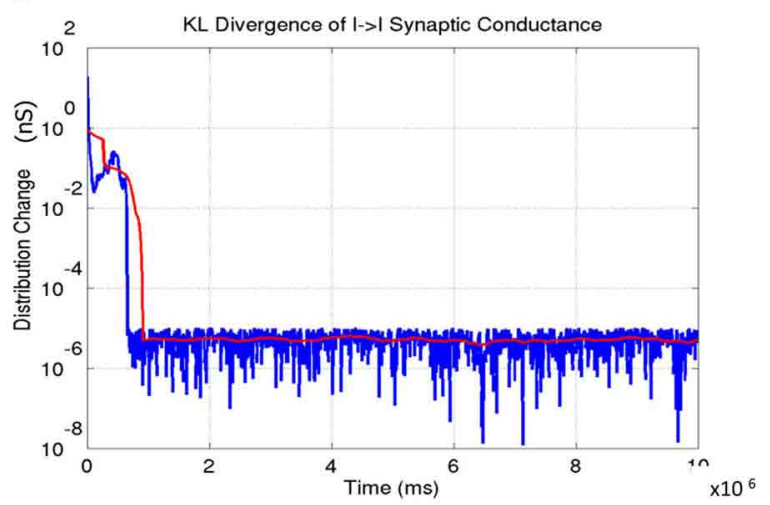

$I \rightarrow I$ change achieve stability very early and remain very stable after indicating that the synaptic conductances between inhibitory interneurons are stabilized rapidly compared to the other three types of synapses. In all the plots, the instantaneous change in synaptic conductance distributions at each time step is shown in blue while the average change is shown in red. et al., 2008; Froemke et al., 2010). It should be noted that in this model, neurons are assumed to be mature from early stages of development. However, in reality there are immature depolarizing neurons in the early stages of development that are characterized by a high concentration of $\mathrm{Cl}^{-}$ions (Hensch, 2005). This aspect of development is not considered in this model.

The model is currently being extended to account for the development of direction selectivity. It is well known that some neurons in the visual cortex are selective to direction of motion of visual stimuli (Weliky et al., 1996; White and Fitzpatrick, 2007). Furthermore, direction selectivity map (DSM) formation appears to lag behind OSM in its formation (Li et al., 2006). Recent physiological evidence suggests that the DSMs in the cortex are nested geometrically within OSMs such that an iso-orientation domain is subdivided into a pair of smaller domains that represent opposite directions of stimulus motion (Kisvarday et al., 2001; White and Fitzpatrick, 2007). Finally, there is also mounting evidence that activitydependent plasticity such as STDP enables the formation of
DSMs (Fiser et al., 2004; Carver et al., 2008; Markram et al., 2011) and recent models show the possibility of forming DSMs using STDP (Buchs and Senn, 2002; Wenisch et al., 2005). Thus a natural extension of the proposed model is to account for the formation of DSMs using STDP within the context of development of all other functional maps such as OSMs and ODMs.

In summary, the present study developed a simple model of a thalamocortical circuit with an initial unstructured map topology that is refined using continuous plasticity in a selforganized fashion to form RCMs, OSMs, and ODMs based on neural activity during three phases of development: endogenously generated cortical activity, followed by activity that arises endogenously in the form of retinal waves and finally activity evoked during sensory experience. Continuous plasticity based on STDP in both excitatory and inhibitory synapses serves as the key mechanism for the development, refinement, and stable maintenance of the formed maps and could also serve as a basis for the development of other functional maps such as DSMs. 
A

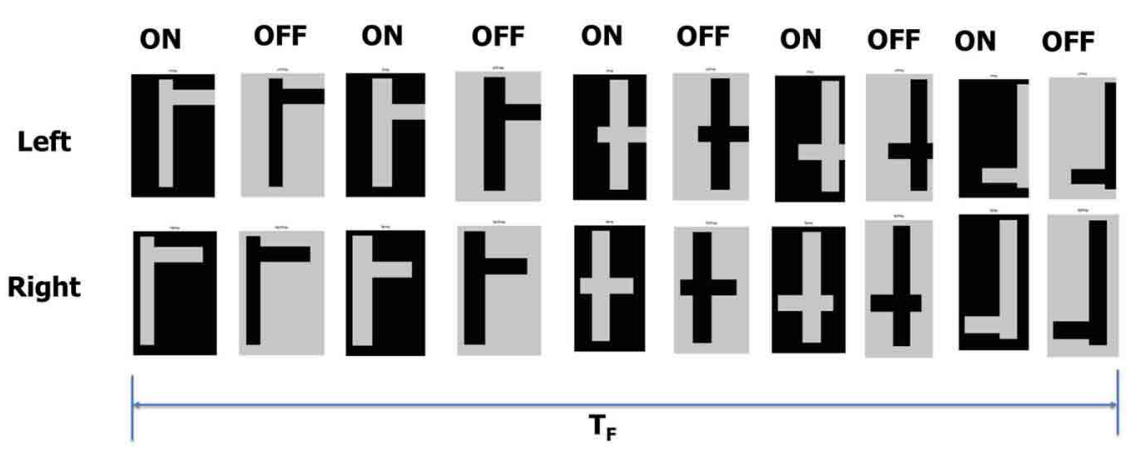

B
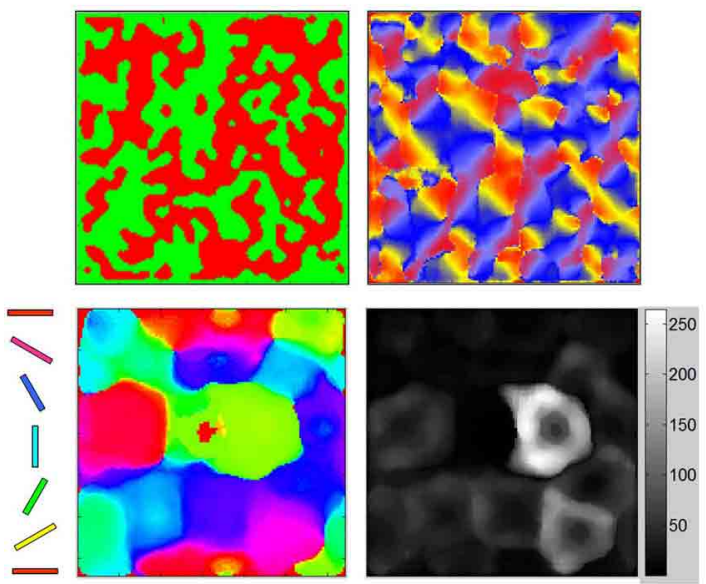

FIGURE 15 |ODM and OSM change in response to new stimuli.

(A) Stability of the development process was tested using a sequence of test patterns shown here as stereo pairs with $\mathrm{ON}$ and OFF images for each pair. The test patterns consist of mixtures of horizontal and vertical lines that combine to form flag-like patterns. These flag-like patterns are presented for total duration of $T_{F}$ seconds. The duration of each flag-like pattern was between 10 and $100 \mathrm{~ms}$. (B) OSM, RCM, and ODM maps after presenting the flag patterns for a duration of $T_{F}=10 \mathrm{~s}$ after Phase 3 shows that the maps are stable (compared to Figure 12) despite constant variations
C
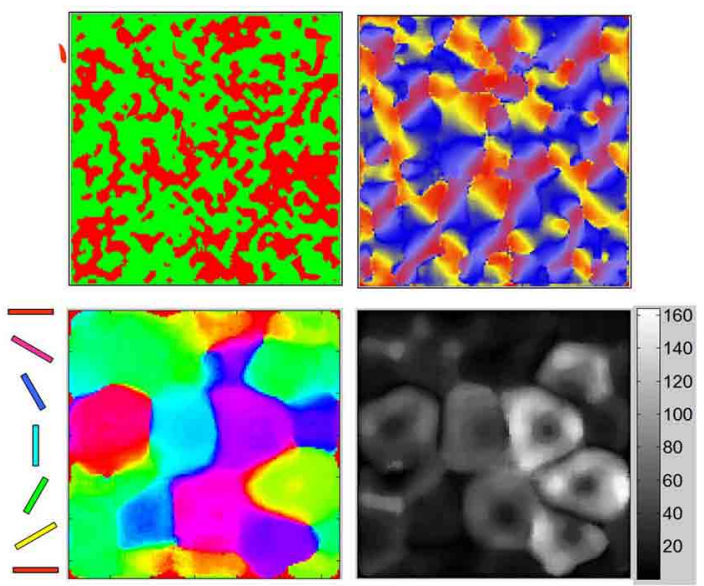

in the duration of presentation of each flag-like patterns. (C) OSM, RCM, and ODM maps after presenting the flag patterns continuously for a duration of $T_{F}=5000 \mathrm{~s}$ after Phase 3 shows that the ODM and OSM change while RCM does not change much at all (compared to panel B). The change in orientation selectivity in the early and late stage of input patterns shows a noticeable change in the OSMs. The number of neurons with responses close to $0^{\circ}$ or $180^{\circ}$ (red and magenta) and $90^{\circ}$ (cyan and green) have gone up compared to the plot in panel (B) indicating that the change reflects the dominance of vertical and horizontal bars in the stimuli.

\section{ACKNOWLEDGMENTS}

The authors acknowledge the support for this work by Defense Advanced Research Projects Agency (DARPA) SyNAPSE contract HRL0011-09-C-001. The views, opinions and/or findings contained in this paper are those of the authors and should not be interpreted as representing the official views or policies, either expressed or implied, of the DARPA or the Department of Defense. We are very grateful for the insightful comments of the reviewers that helped in improving the overall quality of the manuscript.

\section{REFERENCES}

Akerman, C. J., Smyth, D., and Thompson, I. D. (2002). Visual experience before eye-opening and the development of the retinogeniculate pathway. Neuron 36, 869-879.

Antonini, A., and Stryker, M. P. (1978). Plasticity of geniculocortical afferents following brief or prolonged monocular occlusion in the cat. J. Comput. Neurol. 369, 64-82.
Bartsch, A. P., and van Hemmen, J. L. (2000). Combined Hebbian development of geniculocortical and lateral connectivity in a model of primary visual cortex. Biol. Cybern. 84, 41-55.

Basole, A., Kraft-Kerekes, V., White, L. E., and Fitzpatrick, D. (2006). Cortical cartography revisited: a frequency perspective on the functional architecture of visual cortex. Prog. Brain Res. 154, 121-134.
Basole, A., White, L. E., and Fitzpatrick, D. (2003). Mapping multiple stimulus features in the population response of visual cortical neurons. Nature 423, 986-990.

Bauer, C., Burger, T., Stetter, M., and Lang, E. W. (2000). A neural network model for the selforganization of cortical grating cells. Z. Naturforsch. C 55, 282-291.

Bednar, J., and Miikkulainen, R. (2004). Prenatal and postnatal development of laterally connected orientation maps. Neurocomputing 58-60, 985-992.

Bi, Q. Q., and Poo, M. M. (1998). Activity-induced synaptic modification in hippocampal culture, dependence on spike timing, synaptic strength and cell type. J. Neurosci. 18, 10464-10472.

Billings, G., and van Rossum, M. C. W. (2009). Memory retention and spike-timing dependent 
plasticity. J. Neurophysiol. 101, 2755-2788.

Binzegger, T., Douglas, R. J., and Martin, K. A. C. (2004). A quantitative map of the circuit of the cat primary visual cortex. J. Neurosci. 39, 8441-8453.

Blakemore, C., and Cooper, G. F. (1970). Development of brain depends on visual environment. Nature 228, 477-478.

Blasdel, G. G. (1992). Orientation selectivity, preference, and continuity in monkey striate cortex. J. Neurosci. 12, 3139-13161.

Buchs, N. J., and Senn, W. (2002). Spike-based synaptic plasticity and the emergence of direction selective simple cells: simulation results. J. Comput. Neurosci. 13, 167-186.

Buonamano, D., and Merzenich, M. (1998). Cortical plasticity: from synapses to maps. Annu. Rev. Neurosci. 21, 149-156.

Butts, D. (2002). Retinal waves: implications for synaptic learning rules during development. Neuroscientist 8, 243-253.

Caporale, N., and Dan, Y. (2008). Spike timing-dependent plasticity: a Hebbian learning rule. Annu. Rev. Neurosci. 31, 25-46.

Carver, S., Roth, E., Cowan, N. J., and Fortune, E. S. (2008). Synaptic plasticity can produce and enhance direction selectivity. PLoS Comput. Biol. 4:e32. doi: 10.1371/journal.pcbi.0040032

Cassenaer, S., and Laurent, G. (2007). Hebbian STDP in mushroom bodies facilitates the synchronous flow of olfactory information in locusts. Nature 448, 709-713.

Chapman, B., Stryler, M. P., and Bonhoeffer, T. (1996). Development of orientation-preference maps in ferret primary visual cortex. J. Neurosci. 16, 6443-6453.

Chen, C., and Regehr, W. G. (2000). Developmental remodeling of the retinogeniculate synapse. Neuron 28, 955-966.

Chiu, C., and Weliky, M. (2001). Spontaneous activity in developing ferret visual cortex in vivo. J. Neurosci. 21, 8906-8914.

Coppola, D. M., and White, L. E. (2004). Visual experience promotes the isotropic representation of orientation preference. Vis. Neurosci. $21,39-51$.

Crair, M., Gillespie, D., and Stryker, M. (1998). The role of visual experience in the development of columns in cat visual cortex. Science 279, 565-570.

Crair, M., Ruthazer, E., Gillespie, D., and Stryker, M. (1997). Relationship between the ocular dominance and orientation maps in visual cortex of monocularly deprived cats. Neuron 19, 307-318.

Crowley, J. C., and Katz, L. C. (1999) Development of ocular dominance columns in the absence of retinal input. Nat. Neurosci. 2, 1125-1130.

Crowley, J. C., and Katz, L. C. (2002). Ocular dominance development revisited. Curr. Opin. Neurobiol. 12, 104-109.

Debbane, D., Gahwiler, B. H., and Thompson, S. (1998). Longterm synaptic plasticity between pairs of individual CA3 pyramidal cells in rat hippocampal slice cultures. J. Physiol. 507, 237-247.

Fei, L., Fergus, R., and Perona, P. (2006). One-shot learning of object categories. IEEE Trans. Pattern Anal. Mach. Intell. 28, 594-611.

Feller, M. B. (2009). Retinal waves are likely to instruct the formation of eye-specific retinogeniculate projections. Neural Dev. 4, 24-28.

Ferster, D., and Miller, K. D. (2000). Neural mechanisms of orientation selectivity in the visual cortex. Annu. Rev. Neurosci. 23, 441-471.

Finelli, L. A., Haney, S., Bazhenov, M., Stopfer, M., and Sejnowski, T. J. (2008). Synaptic learning rules and sparse coding in a model sensory system. PLoS Comput. Biol. 4:e1000062. doi: 10.1371/journal.pcbi.1000062

Fiser, J., Chiu, C., and Weliky, M. (2004). Small modulation of ongoing cortical dynamics of sensory input during natural vision. Nature $431,573-578$.

Froemke, R. C., Letzkus, J. J., Kampa, B. M., Hang, G. B., and Stuart, G. J. (2010). Dendritic synapse location and neocortical spiketiming dependent plasticity. Fron. Syn. Neurosci. 2:29. doi: 10.3389/fnsyn.2010.00029

Godfrey, K. B., and Swindale, N. V. (2007). Retinal wave behavior through activitydependent refractory periods. PLoS Comput. Biol. 3:e245. doi: 10.1371/journal.pcbi.0030245

Hartmann, K., Bruehl, C., Golovko, T., and Draguhn, A. (2008). Fast homeostatic plasticity of inhibition via activity-dependent vesicular filling. PLoS ONE 3:e2979. doi: 10.1371/journal.pone.0002979

Hensch, T. K. (2005). Critical period plasticity in local cortical circuits. Nat. Neurosci. 6, 877-888.

Hodgkin, A. L., and Huxley, A. F. (1952). A quantitative description of membrane current and application to conduction and excitation in nerve. J. Physiol. 117, 500-544.

Hubel, D., and Wiesel, T. N. (1962) Receptive fields, binocular interaction and functional architecture in the cat's visual cortex. J. Physiol. 160, 106-154.

Hubel, D., and Wiesel, T. N. (2005) Brain and Visual Perception. New York, NY: Oxford University Press.

Hubel, D. H., and Wiesel, T. N. (1963) Receptive fields of striate cortex of very young, visually inexperienced kittens. J. Neurophysiol. 26 994-1002.

Hubel, D. H., and Wiesel, T. N. (1968). Receptive fields and functional architecture of monkey striate cortex. J. Physiol. 195, 215-243.

Huberman, A. D., Feller, M. B., and Chapman, B. (2008). Mechanisms underlying development of visual maps and receptive fields. Annu. Rev. Neurosci. 31, 479-509.

Huberman, A. D., Speer, C. M., and Chapman, B. (2006). Spontaneous retinal activity mediates development of ocular dominance columns and binocular receptive fields in V1. Neuron 52, 247-254.

Izhikevich, E. (2004). Which model to use for cortical spiking neurons? IEEE Trans. Neural Netw. 15, 1063-1070.

Jacob, V., Brasier, D. J., Erchova, I., Feldman, D., and Shulz, D. E. (2007). Spike timing-dependent synaptic depression in the in vivo barrel cortex of the rat. J. Neurosci. 27, 1271-1284.

Kang, K., Shelley, M., and Sompolinsky, H. (2003). Mexican hats and pinwheels in visual cortex. Proc. Natl. Acad. Sci. U.S.A. 100, 2848-2853.

Katz, L., and Shatz, C. (1996). Synaptic activity and the construction of cortical circuits. Science 274, 1133-1138.

Katz, L. C., and Crowley, J. C. (2002). Development of cortical circuits: lessons from ocular dominance columns. Nat. Rev. Neurosci. 3, 34-42.

Kisvarday, Z. F., Buzas, P., and Eysel, U. T. (2001). Calculating direction maps from intrinsic signals revealed by optical imaging. Cereb. Cortex 11 , 636-647.

Koehler, C. L., Akimov, N. P., and Renteria, R. C. (2011). Receptive field center size decreases and firing properties mature in $\mathrm{ON}$ and $\mathrm{OFF}$ retinal ganglion cells after eye opening in the mouse. J. Neurophysiol. $106,895-904$.
Krelle, A. K., Bonhoeffer, T., and Hubener, M. (2011). Altered visual experience induces instructive changes of orientation preference in mouse visual cortex. J. Neurosci. 31, 13911-13920.

Kremkow, J., Kumar, A., Rotter, S., and Aertsen, A. (2007). Emergence of population synchrony in a layered network of the cat visual cortex. Neurocomputing 70, 2069-2073.

Kullback, S. (1987). The kullbackleibler distance. Am. Stat. 41, 340-341.

Kumar, A., Schraeder, S., and Aertsen, A. (2008). High conductance state of cortical networks. Neural Comput. 20, 1-43.

Levy, W. B., and Steward, D. (1983). Temporal contiguity requirements for long-term associative potentiation/depression in the hippocampus. Neuroscience 8 791-797.

Li, Y., Fitzpatrick, D., and White, L. E. (2006). The development of direction selectivity in ferret visual cortex requires early visual experience. Nat. Neurosci. 9, 676-681.

Magee, J. C., and Johnston, D. (1997). A synaptically controlled, associative signal for Hebbian plasticity in hippocampal neurons. Science 275 , 209-213.

Maldonado, P. E., Godecke, I., Gray, C. M., and Bonhoeffer, T. (1997). Orientation selectivity in pinwheel centers in cat striate cortex. Science 276, 1551-1555.

Mark, S., and Tsodyks, M. (2012). Population spikes in cortical networks during different functional states. Front. Comput. Neurosci. 6:43. doi: 10.3389/fncom. 2012.00043

Markram, H., Gerstner, W., and Sjostrom, P. J. (2011). A history of spike-timing-dependent plasticity. Front. Synaptic Neurosci. 3:4. doi: 10.3389/fnsyn.2011.00004

Markram, H., Lubke, J., Frotscher, M., and Sakmann, B. (1997). Regulation of synaptic efficacy by coincidence of postsynaptic APs and EPSPs. Science 275, 213-215.

Meliza, C. D., and Dan, Y. (2006). Receptive-field modification in rat visual cortex induced by paired visual stimulation and single-cell spiking. Neuron 49, 183-189.

Miikkulainen, R., Bednar, J. A., Choe, Y., and Shirosh, J. (2005). Computational Maps in the Visual Cortex. New York, NY: Springer.

Miller, K. (1996). "Receptive fields and maps in the visual cortex: model 
of ocular dominance and orientation columns," in Models of Neural Networks, III, eds E. Domany, J. van Hemmen, and K. Schulten (New York, NY: Springer-Verlag), 55-78.

Miller, K. D., Erwin, E., and Kayser, A. (1999). Is the development of orientation selectivity instructed by activity? J. Neurobiol. 41, 44-57.

Miller, K. D., Keller, J. B., and Stryker, M. P. (1989). Ocular dominance column development: analysis and simulation. Science 245, 605-615.

Mooney, R., Penn, A. A., Gallego, R., and Shatz, C. J. (1996). Thalamic relay of spontaneous retinal activity prior to vision. Neuron 17, 863-874.

Mu, Y., and Poo, M. M. (2006). Spike timing-dependent LTP/LTD mediates visual experiencedependent plasticity in a developing retinotectal system. Neuron 50, 115-125.

Myhr, K. L., Lukasiewicz, P. D., and Wong, R. O. L. (2001). Mechanisms underlying developmental changes in the firing patterns of $\mathrm{ON}$ and OFF retinal ganglion cells during refinement of their central projections. J. Neurosci. 21, 8664-8671.

Nageswaran, J. M., Dutt, N., Krichmar, J. L., Nicolau, A., and Veidenbaum, A. V. (2009). A configurable simulation environment for the efficient simulation of large-scale spiking neural networks on graphics processors. Neural Netw. 22, 791-800.

Ohki, K., Chung, S., Kara, P., Hubener, M., Bonhoeffer, T., and Reid, R. C. (2006). Highly ordered arrangement of single neurons in orientation pinwheels. Nature 442, 925-928.

Okun, M., and Lampl, I. (2008). Instantaneous correlation of excitation and inhibition during ongoing and sensory-evoked activities. Nat. Neurosci. 11, 535-537.

Perin, R., Berger, T. K., and Markram, H. (2011). A synaptic organizing principle for cortical neuronal groups. Proc. Natl. Acad. Sci. U.S.A. 108, 5419-5424.

Pouille, F., Burgin, A. M., Adesnik, H., Atallah, B. V., and Scanziani, M. (2009). Input normalization by global feedforward inhibition expands cortical dynamic range. Nat. Neurosci. 12, 1577-1585.

Purves, D., and Lichtman, J. (1985). Principles of Neural Development. Sunderland, MA: Sinauer Associates.
Rao, S. C., Toth, L. J., and Sur, M. (1997). Optical imaged maps of orientation preference in primary visual cortex of cats and ferrets. J. Comput. Neurol. 387, 358-370.

Ruthazer, E., and Stryker, M. (1996). The role of activity in the development of long-range horizontal connections in area 17 of the ferret. J. Neurosci. 18, 7253-7269.

Schiller, P. H. (1992). The ON and OFF channels of the visual system. Trends Neurosci. 15, 86-92.

Schummers, J., Marino, J., and Sur, M. (2004). Local networks in visual cortex and their influence on neuronal responses and dynamics. J. Physiol. 98, 429-441.

Schummers, J., Sharma, J., and Sur, M. (2005). Bottom-up and top-down dynamics in visual cortex. Prog. Brain Res. 149, 65-81.

Sengpiel, F., Stawinski, P., and Bonhoeffer, T. (1999). Influence of experience on orientation maps in cat visual cortex. Nat. Neurosci. 2, 727-732.

Shapley, R., Hawken, M., and Ringach, D. L. (2003). Dynamics of orientation selectivity in the primary visual cortex and the importance of cortical inhibition. Neuron 38, 689-699.

Shapley, R., Hawken, M., and Xing, D. (2007). The dynamics of visual responses in the primary visual cortex. Prog. Brain Res. 165, 21-32.

Sirosh, J., and Miikkulainen, R. (1995). Self-Organization and Functional Role of Lateral Connections and Multisize Receptive Fields in the Primary Visual Cortex. University of Texas at Austin, Technical Report AI95-230, May 1995.

Sjostrom, P. J., Rancz, E. A., Roth, A., and Hausser, M. (2008). Dendritic excitability and synaptic plasticity. Physiol. Rev. 88, 769-840.

Smith, S. L., and Trachtenberg, J. T. (2007). Experience-dependent binocular competition in the visual cortex begins at eye opening. Nat. Neurosci. 10, 370-375.

Somers, D. C., Nelson, S. B., and Sur, M. (1995). An emergent model of orientation selectivity in cat visual cortical simple cells. J. Neurosci. 15, 773-786.

Song, S., and Abbott, L. F. (2001). Cortical remapping through spike timing-dependent plasticity. Neuron $32,1-20$.

Song, S., Miller, K., and Abbott, L. F. (2000). Competitive Hebbian learning through spike timingdependent plasticity. Nat. Neurosci. 3, 919-925.
Song, S., Sjostrom, P. J., Reigl, M., Nelson, S., and Chklovskii, D. B. (2005). Highly nonrandom features of synaotic connectivity in local cortical circuits. PLoS Biol. 3:e68. doi: 10.1371/journal.pbio.0030068

Swindale, N. V. (2000). How many maps are there in visual cortex? Cereb. Cortex 10, 633-643.

Taha, S. A., and Stryker, M. P. (2005). Molecular substrates of plasticity in the developing visual cortex. Prog. Brain Res. 147, 101-114.

Trachtenberg, J. T., Trepel, C., and Stryker, M. P. (2000). Rapid extragranular plasticity in the absence of thalamocortical plasticity in the developing visual cortex. Science 287, 2029-2032.

Tsodyks, M., and Markram, H. (1997). The neural code between neocortical pyramidal neurons depends on neurotransmitter release probability. Proc. Natl. Acad. Sci. U.S.A. 94, 719-723.

Tsodyks, M., Pawelzik, K., and Markram, H. (1998). Neural networks with dynamic synapses. Neural Comput. 10, 821-835.

Turrigiano, G. G., and Nelson, S. B (2004). Homeostatic plasticity in the developing nervous system. Nat. Rev. Neurosci. 5, 97-107.

Ursey, W. M., Reppan, J. B., and Reid, R. C. (1999). Specificity and strength of retinogeniculate connections. J. Neurophysiol. 82, 3527-3540.

Vislay-Meltzer, R. L., Kampff, A. R., and Engert, F. (2006). Spatiotemporal specificity of neuronal activity directs the modification of receptive fields in the developing retinotectal system. Neuron 50, 101-114.

Vogels, T. P., Rajan, K., and Abbott, L. F. (2005). Neural network dynamics. Annu. Rev. Neurosci. 28, 357-376.

Vogels, T. P., Sprekeler, H., Zenke, F., Clopath, C., and Gerstner W. (2011). Inhibitory plasticity balances excitation and inhibition in sensory pathways and memory networks. Science 334 1569-1573.

Voges, N., and Perrinet, L. (2012). Complete dynamics in recurrent cortical networks based on spatially realistic connectivities. Front. Comput. Neurosci. 6:41. doi: 10.3389/fncom.2012.00041

Watt, A. J., and Desai, N. S. (2010). Homeostatic plasticity and STDP: keeping a neuron's cool in a fluctuating world. Front. Synaptic Neurosci. 2:5. doi: 10.3389/fnsyn.2010.00005
Weliky, M., Bosking, W. H., and Fitzpatrick, D. (1996). A systematic map of direction preference in primary visual cortex. Nature 379, 725-728.

Wenisch, O. G., Noll, J., and van Hemmen, J. L. (2005). Spontaneously emerging direction selectivity maps in visual cortex through STDP. Biol. Cybern. 93, 239-247.

White, L. E., Coppola, D. M., and Fitzpatrick, D. (2001). The contribution of sensory experience to the maturation of orientation selectivity in ferret visual cortex. Nature 411 1049-1052.

White, L. E., and Fitzpatrick, D. (2007). Vision and cortical map development. Neuron 56, 327-338.

Wiesel, T. N., and Hubel, D. H. (1974). Ordered arrangement of orientation columns in monkeys lacking visual experience. J. Comput. Neurol. 158, 307-318.

Williams, S. R., Wozny, C., and Mitchell, S. J. (2007). The back and forth of dendritic plasticity. Neuron 56, 947-953.

Wong, O. L. R. (1999). Retinal waves and visual system development, Annu. Rev. Neurosci. 22, 29-47.

Woodin, M. A., Ganguly, K., and Poo, M. M. (2003). Coincident preand postsynaptic activity modifies GABAergic synapses by postsynaptic changes in $\mathrm{Cl}^{-}$transporter activity. Neuron $39,807-820$.

Xing, D., Ringach, D. L., Hawken, M. J., and Shapley, R. M. (2011). The contribution of untuned suppression to orientation selectivity in macaque V1. J. Neurosci. 31, 15972-15982.

Yang, Q., Qi, X., and Wang, Y. (2012). A neural network model on selforganizing emergence of simplecell receptive field with orientation selectivity in visual cortex. Sci. China C Life Sci. 44, 469-478.

Yao, H., and Dan, Y. (2001). Stimulus timing-dependent plasticity in cortical processing of orientation. Neuron 32, 315-323.

Young, J. M., Waleszcyk, W. J., Wang, C., Calford, M. B., Dreher, B., and Obermayer, K. (2007) Cortical reorganization consistent with spike timing but not correlation-dependent plasticity Nat. Neurosci. 10, 887-895.

Yu, H., Farley, B. J., Jin, D. Z., and Sur, M. (2005). The coordinated mapping of visual space and response features in visual cortex. Neuron 47 , 267-280.

Yuste, R., and Sur, M. (1999). Development and plasticity of the cerebral cortex: from molecules to maps. J. Neurosci. 41, 1-6. 
Zaghloul, K. A., Boahen, K., and Demb, J. B. (2003). Different circuits for $\mathrm{ON}$ and $\mathrm{OFF}$ retinal ganglion cells cause different contrast sensitivities. J. Neurosci. 23, 2645-2654.

Zhang, Z., and Xu, G. (1998). A unified theory of uncalibrated stereo for both perspective and affine cameras. J. Math. Imaging Vis. 9, 213-229.

Conflict of Interest Statement: The authors declare that the research was conducted in the absence of any commercial or financial relationships that could be construed as a potential conflict of interest.
Received: 05 August 2012; accepted: 09 February 2013; published online: 27 February 2013.

Citation: Srinivasa $N$ and Jiang $Q$ (2013) Stable learning of functional maps in self-organizing spiking neural networks with continuous synaptic plasticity. Front. Comput. Neurosci. 7:10. doi: 10.3389/fncom.2013.00010
Copyright (c) 2013 Srinivasa and Jiang This is an open-access article distributed under the terms of the Creative Commons Attribution License, which permits use, distribution and reproduction in other forums, provided the original authors and source are credited and subject to any copyright notices concerning any third-party graphics etc. 